\title{
Checklist of the millipedes (Myriapoda: Diplopoda) of the Republic of North Macedonia
}

\author{
Контрольный список диплопод (Myriapoda: Diplopoda) \\ Республики Северная Македония
}

\section{Zvezdana S. Jovanović ${ }^{1 *}$, Aleksandra Cvetkovska-Gjorgjievska ${ }^{2}$, Dana Prelić $^{2}$, Dragan Ž. Antić ${ }^{1,3}$, Slobodan E. Makarov ${ }^{1,3}$ Звездана С. Јовановић ${ }^{1 *}$, Александра Цветковска-Ђорђиевска ${ }^{2}$, Аана Прелић², Араган Ж. Антић ${ }^{1,3}$, Слободан Е. Макаров ${ }^{1,3}$}

\footnotetext{
${ }^{1}$ Institute of Zoology, Faculty of Biology, University of Belgrade, Studentski Trg 16, 11000 Belgrade, Serbia; E-mail: zjovanovic@bio.bg.ac.rs ${ }^{2}$ Institute of Biology, Faculty of Natural Sciences and Mathematics, Saints Cyril and Methodius University, Arhimedova 3, 1000 Skopje, North Macedonia

${ }^{3}$ Serbian Biospeleological Society, Trg Dositeja Obradoviæa 2, 21000 Novi Sad, Serbia
}

KEY WORDS: Diplopoda, diversity, fauna, North Macedonia, Balkan Peninsula.

КЛЮЧЕВЫЕ СЛОВА: Diplopoda, разнообразие, фауна, Северная Македония, Балканский полуостров.

ABSTRACT. The millipede fauna of the Republic of North Macedonia presently includes 67 species from 33 genera, 15 families and seven orders. As many as 12 and 19 species, or ca. $18 \%$ and $28 \%$ of all records, are endemic to North Macedonia and the Balkan Peninsula, respectively. The most speciose families are Julidae, with 29 species, or $43 \%$, followed by Polydesmidae with 16 species, or 24\%. Hirudisoma hirsutum (Verhoeff, 1901) and Brachydesmus cristofer Strasser, 1966 are new to the millipede fauna of North Macedonia. The distribution pattern and maps are given for each species.

How to cite this article: Jovanović Z.S., Cvetkovska-Gjorgjievska A., Prelić D., Antić D.Ž., Makarov S.E. 2019. Checklist of the millipedes (Myriapoda: Diplopoda) of the Republic of North Macedonia // Arthropoda Selecta. Vol.28. No.2. P.191-205. doi: 10.15298/arthsel. 28.2.02

РЕЗЮМЕ. Диплоподы фауны Республики Северная Македония ныне всключают 67 видов из 33 родов, 15 семейств и семи отрядов. Из них 12 и 19 видов, или примерно $18 \%$ и $28 \%$ всех находок, эндемики соответственно Северной Македонии и Балканского полуострова. Самые богатые видами семейства - Julidae с 29 видами, или 43\% фауны, и Polydesmidae с 16 видами, или 24\%. Виды Hirudisoma hirsutum (Verhoeff, 1901) и Brachydesmus cristofer Strasser, 1966 - новые для фауны Македонии. Для каждого вида представлены тип ареала и карта с распространением.

\section{Introduction}

The Republic of North Macedonia occupies the central part of the Balkan Peninsula. It has varied relief structure and age, geology and soil types, a situation resulting from its complex geotectonic evolution, which shaped the republic's present-day rich biodiversity. The mountains of the older Rhodopian tectonic mass (Mts Belasica and Osogovo) and those of the younger Dinaric tectonic mass (Šar Planina Mountains and Mts Bistra, Korab and Pelister) include localities with a large number of reported species. The shores of the large tectonic lakes Ohrid and Prespa and of the smaller Lake Dojran are also the sites of numerous millipede records. Different influences, such as the presence of various water bodies, the existence of high mountains and human impacts, modify the mostly continental and Mediterranean climate. Vegetation is represented by forest, grass, semi-steppe and marsh communities, together with ones of certain other types as well. This diversity of natural conditions contributes to the profound species richness of the republic's flora and fauna [Makarov et al., 2004].

Diplopoda are ancient terrestrial arthropods with more than 12,000 described species, while their diversity is estimated to range between ca. 25,000 to 80,000 species [Hopkin, Read, 1992; Golovatch, Kime, 2009; Sierwald, Bond, 2007]. One of the globe's most important centres of millipede diversity is the Balkan Peninsula, which is inhabited by almost 1,000 species, including many endemic taxa. The first comprehensive review of the millipede fauna of present-day North Macedonia was published by Strasser [1971a, b], pre- 


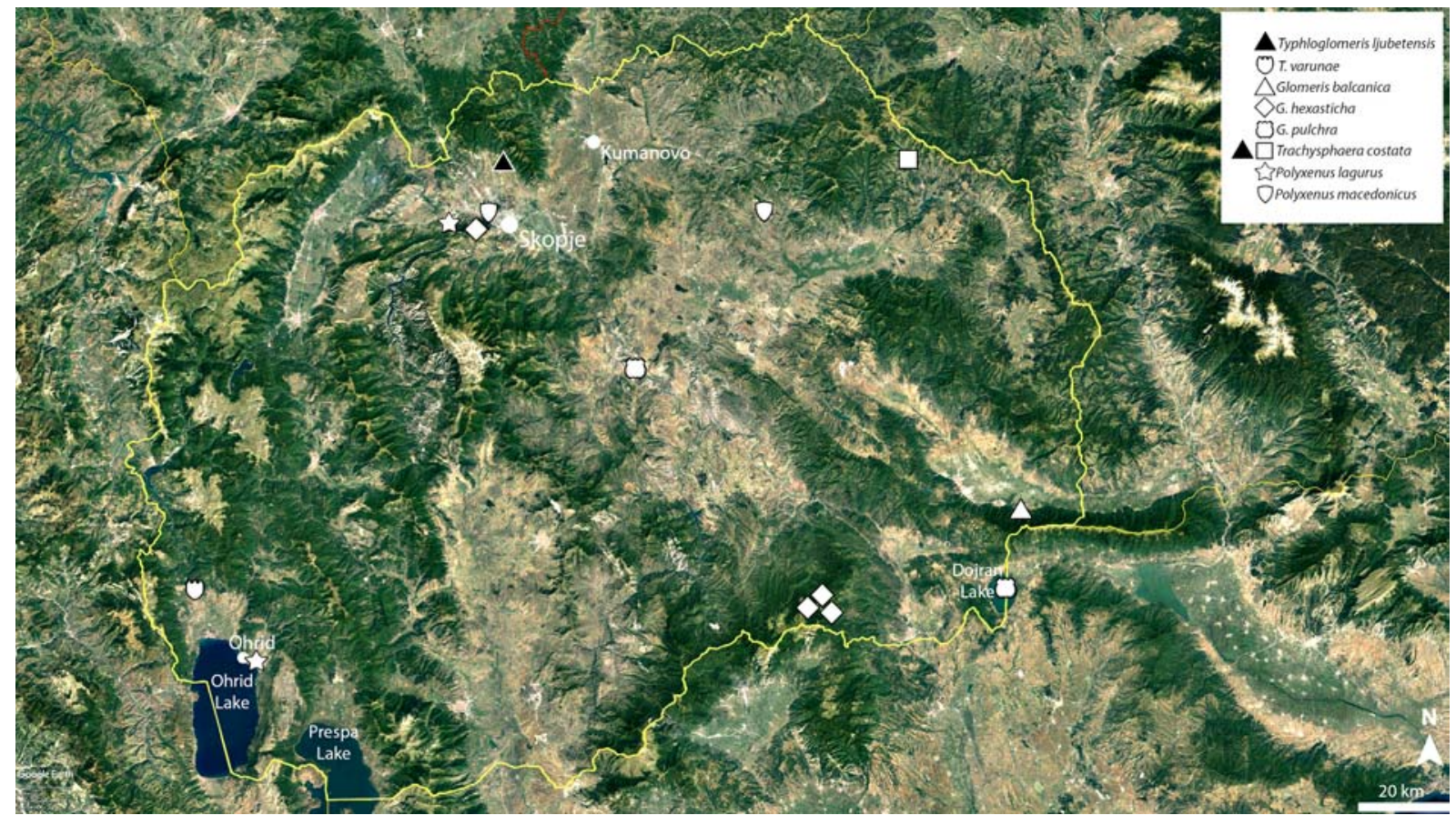

Fig. 1. Distribution of representatives of the orders Glomerida and Polyxenida in North Macedonia.

Рис. 1. Распространение представителей отрядов Glomerida и Polyxenida в Северной Македонии.

ceded by the studies by Attems [1929, 1959], and it listed already 43 species or subspecies. Research efforts carried out in the late 1980's and early 1990's supplemented these records with a number of new taxa, thereby resulting in 59 species or subspecies, as reported by Mršić [1993a]. In the late 1990's and early 2000's, Ćurčić et al. [1999a, b, 2002a, b] and Makarov et al. [2001, 2003] added several new species to the diplopod fauna of North Macedonia. Makarov et al. [2004] recognized 61 species, 12 of which were endemic to the country. Finally, Petkovski [2009] published his National Catalogue containing 62 millipede species. Kime \& Enghoff [2011, 2017], in their panEuropean atlases, listed most of the millipede species described, accepted or recorded to date from North Macedonia, except for those in the order Chordeumatida. In their comprehensive work, Vagalinski \& Lazányi [2018] revised the tribe Brachyiulini, including North Macedonian species.

The objective of the present study is to critically list all members of the North Macedonian diplopod fauna based on published records and new material collected between the 1990's and late 2010's. The synonymy lists are largely after Kime \& Enghoff [2011, 2017], Spelda [2013], and Vagalinski \& Lazányi [2018].

\section{Material and methods}

The specimens collected were examined using a Nikon SMZ 745T stereo microscope and are presently deposited at the Institute of Zoology, Faculty of Biology, University of Belgrade, Serbia. All taxonomically important structures were dissected, mounted in glycerine as temporary microscope preparations, and observed with a Carl Zeiss Axioscope 40 microscope. The distribution maps were created using Google Earth Pro (version 7.3.0.3832) and Adobe Photoshop CS6.

\section{List of species}

Order POLYXENIDA

Family POLYXENIDAE

\section{Polyxenus lagurus (Linnaeus, 1758)}

Scolopendra lagurus Linnaeus, 1758

Polyxenus germanicus Verhoeff, 1941

Distribution in the Republic of North Macedonia: Ohrid [Makarov et al., 2004]; river Treska, Skopje [Attems, 1929]; without precise locality [Strasser 1971a, b; Mršić, 1993a; Mauriès et al., 1997] (Fig. 1).

World distribution: Albania, Austria, Azores Islands, Balearic Islands, Belarus, Belgium, Bulgaria, Channel Islands, Corsica, Croatia, Cyclades Islands, Czech Republic, Germany, Danish mainland, Estonia, European Turkey (without islands in the Sea of Marmara), French mainland, Great Britain, Hungary, Ireland, Italian mainland, Latvia, Lithuania, Luxembourg, Republic of North Macedonia, Moldova, Northern Ireland, Norwegian mainland and inshore islands, Poland, Portuguese mainland, Romania, Russia (Central European Russia, North European Russia, Northwest Russia), Sicily and neighbouring Italian islands, Spanish mainland, Serbia, Slovenia, Slovakia, Sweden, Switzerland, the Netherlands, Ukraine, and Northern USA [Kime, Enghoff, 2011]. 


\section{Polyxenus macedonicus Verhoeff, 1952}

Distribution in the Republic of North Macedonia: Skopje [Verhoeff, 1952; Strasser, 1971a]; village of Strmoš, Probištip [Mršić, 1993a]; without precise locality [Mauriès et al., 1997; Ćurčić et al., 2002a; Makarov et al., 2004] (Fig. 1).

World distribution: Corsica, French mainland, Republic of North Macedonia, Malta [Kime, Enghoff, 2011].

\section{Order GLOMERIDA \\ Family GLOMERIDELLIDAE}

\section{Typhloglomeris ljubetensis (Attems, 1929)**}

Albanoglomus ljubetensis Attems, 1929

Albanoglomus ljubotensis Mršić, 1985

Distribution in the Republic of North Macedonia: Ljuboten, Šar Planina Mts [Attems, 1929; Strasser, 1971a; Mršić, 1993a; Makarov et al., 2003, 2004]; without precise locality [Strasser, 1971b; Mauriès et al., 1997; Ćurčić et al., 1999a, 2000] (Fig. 1).

World distribution: Republic of North Macedonia, Serbia [Kime, Enghoff, 2011].

Balkan endemic.

\section{Typhloglomeris varunae Makarov, Lučić,} Tomić et Karaman, 2003**

MATERIAL. $1 \sigma^{r}, 1$, and 3 juvenile topotypes: Mlečnik Cave, village of Tašmarunište, Struga, 21.IV.2006, leg. I. Karaman.

Distribution in the Republic of North Macedonia: Mlečnik Cave, village of Tašmarunište, Struga [Makarov et al., 2003, 2004; present study] (Fig. 1).

North Macedonian endemic.

\section{Family GLOMERIDAE}

\section{Glomeris balcanica Verhoeff, 1906**}

Glomeris bureschi Verhoeff, 1926

Glomeris latemarginata Attems, 1927

Glomeris hahni Lang, 1935

Glomeris kaloferensis Verhoeff, 1937

Glomeris olympiaca Verhoeff, 1937

Glomeris rhodopina Verhoeff, 1937

Glomeris sakarensis Verhoeff, 1937

MATERIAL. 1 , near the village of Popadija, Mt Belasica, $767 \mathrm{~m}$ a.s.1., Quercus-Fraxinus forest, 24.IV.2010; 1 ㅇ, same locality, $1200 \mathrm{~m}$ a.s.1., Calamintha-Fagus community, 24.IV.2010; $8 \sigma^{7} \sigma^{7}, 8$ 우, same locality, $847 \mathrm{~m}$ a.s.1., Quercus-Fraxinus forest, 28.V.2010; $13 \sigma^{7} \sigma^{\top}, 4$ क्ष, same locality, $1100 \mathrm{~m}$ a.s.1., FestucaFagus community, 28.V.2010; $11 \bigcirc^{\top} \sigma^{7}, 1$ ㅇ, same locality, $1200 \mathrm{~m}$ a.s.1., Calamintha-Fagus community, 28.V.2010; $4 \sigma^{\top} \sigma^{\top}, 1$ 웅 same locality, 847 m a.s.1., Quercus-Fraxinus forest, 3.VII.2010; 1 $\mathrm{O}^{7}$, same locality, $1038 \mathrm{~m}$ a.s.1., Quercus-Fraxinus forest, 3.VII 2010; 3 +o , same locality, $1100 \mathrm{~m}$ a.s.1., Festuca-Fagus community, 3.VII.2010; $1 \sigma^{7}$, same locality, $1200 \mathrm{~m}$ a.s.1., CalaminthaFagus community, 3.VII.2010; 1 ð', between Pod and Suva Èešma, Mt Belasica, $587 \mathrm{~m}$ a.s.1., Quercus-Carpinus forest, 28.V.2010; $6 \sigma^{7} \sigma^{7}, 1$ ㅇ, 1 juv., near Groba, Mt Belasica, $1300 \mathrm{~m}$ a.s.1., Calamintha-Fagus community, 28.V.2010; 1 \% , 1 क, same locality, $1300 \mathrm{~m}$ a.s.1., Calamintha-Fagus community, 3.VII.2010; 1 , same locality, $1300 \mathrm{~m}$ a.s.l., Calamintha-Fagus community, 4.IX.2010; $1 \sigma^{7}, 1$ \%, near Pisana Skala slopes, Mt Belasica, 1385 $\mathrm{m}$ a.s.1., Calamintha-Fagus community, 28.V.2010; $3 \sigma^{\top} \sigma^{7}, 4$, 40 , same locality, $1442 \mathrm{~m}$ a.s.1., cleared area, 28.V.2010; $2 \sigma^{\top} \sigma^{\top}, 1$, same locality, $1385 \mathrm{~m}$ a.s.1., Calamintha-Fagus community, 3.VII. 2010; 1 ऽ, 5 우, same locality, $1442 \mathrm{~m}$ a.s.1., cleared area, 3.VII. 2010; 1 juv., same locality, $1385 \mathrm{~m}$ a.s.1., Calamintha-Fagus community, 4.IX.2010; 1 q, same locality, $1442 \mathrm{~m}$ a.s.1., cleared area, 4.IX.2010, all leg. A.C. Gjorgjievska.

Distribution in the Republic of North Macedonia: Mt Belasica (present study); village of Kosturino [Mršić, 1993a; Makarov et al., 2004]; without precise locality [Mauriès et al., 1997] (Fig. 1).

World distribution: Bulgaria, European Turkey (without islands in the Sea of Marmara), Greek mainland, and the Republic of North Macedonia [Kime, Enghoff, 2011].

Balkan endemic.

\section{Glomeris hexasticha Brandt, 1833}

Glomeris mniszechii Nowicki, 1870

Glomeris ambigua Haase, 1886

Glomeris ruscorum Verhoeff, 1929

Glomeris chiemensis Verhoeff, 1941

MATERIAL. 1 + , Mt Kožuf, $550 \mathrm{~m}$ a.s.1., Quercus-fraxinus forest, 15.VI.2010; 1 ㅇ, Mt Kožuf, Gorska Buka, 1505 m a.s.1., Calamintha-Fagus community, 14.VI.2016; 1 ㅇ, Mt Kožuf, ecotope between spruce forests, $1673 \mathrm{~m}$ a.s.1., 19.VII.2016, all leg. D. Matevski; 1 \%, Mt Vodno, Skopje, 770 m a.s.1., Mediterranean pine forest, 15.VI. 2015, leg. R. Blaževska.

Distribution in the Republic of North Macedonia: Belčište-Blato [Mršić, 1993a]; village of Dubrovnica [Mršić, 1993]; Mt Konečka [Mršić, 1993]; Mt Kožuf (present study); Matka [Mršić, 1993a; Ćurčić et al., 1999a]; Mt Osogovo [Mršić, 1993a]; Popova Šapka, Šar Planina Mts [Mršić, 1993a]; village of Rašče, near Skopje [Ćurčić et al., 1999a; Makarov et al., 2004]; Lake Tikveš [Mršić, 1993a]; village of Treska, Skopje [Attems, 1929; Mršić, 1993a]; Mt Vodno near Skopje [Mršić, 1993a; present study]; without precise locality [Strasser, 1971a, b; Mauriès et al., 1997] (Fig. 1).

World distribution: Albania, Asiatic Turkey, Austria, Bosnia and Herzegovina, Bulgaria, Croatia, Czech Republic, European Turkey (without islands in the Sea of Marmara), Germany, Hungary, Italian mainland, Republic of North Macedonia, Moldova, Montenegro, Poland, Romania, Russia (Central European), Serbia, Slovenia, Slovakia, Switzerland, and Ukraine [Kime, Enghoff, 2011].

\section{Glomeris pulchra C. L. Koch, 1847}

Glomeris buccariensis Verhoeff, 1924

Glomeris quarnerona Attems, 1927

Glomeris kratochvili Lang, 1939

Distribution in the Republic of North Macedonia: Mt Babuna [Mršić, 1993a]; Lake Dojran [Mršić, 1993a; Makarov et al., 2004] (Fig. 1).

World distribution: Albania, Bosnia and Herzegovina, Croatia, Italian mainland, Republic of North Macedonia, and Montenegro [Kime, Enghoff, 2011].

\section{Trachysphaera costata (Waga, 1857)}

\section{Gervaisia costata Waga, 1857}

Trachysphaera hyrtlii Wankel, 1861

Gervaisia rotundata Lignau, 1911

Trachysphaera asiaeminoris (Verhoeff, 1940)

Gervaisia pigmentifera Verhoeff, 1941

Gervaisia similicostata Radu, Ceuca, 1951

Distribution in the Republic of North Macedonia: Ljuboten, Šar Planina Mts [Attems, 1929]; Rudnik, Strumica [Mršić, 1993a]; Sasa [Mršić, 1993a]; without precise 


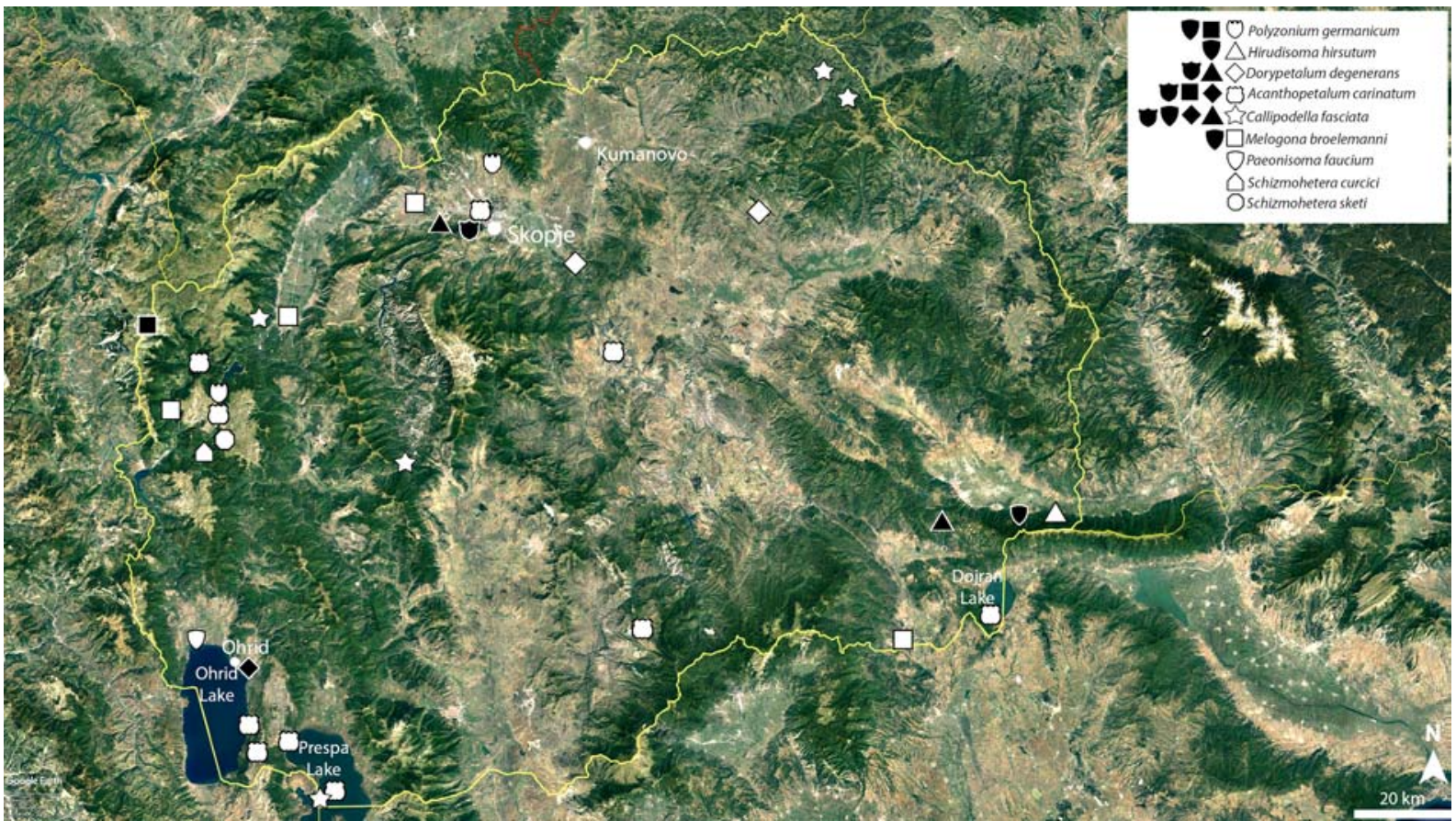

Fig. 2. Distribution of representatives of the orders Polyzoniida, Callipodida and Chordeumatida in North Macedonia.

Рис. 2. Распространение представителей отрядов Polyzoniida, Callipodida и Chordeumatida в Северной Македонии.

locality [Strasser, 1971a, b; Mauriès et al., 1997; Makarov et al., 2004] (Fig. 1).

World distribution: Bosnia and Herzegovina, Bulgaria, Croatia, Czech Republic, Germany, Greek mainland with Evia Island, Hungary, Republic of North Macedonia, Montenegro, Poland, Romania, Serbia, Slovenia, Slovakia, Ukraine, and possibly Albania [Kime, Enghoff, 2011].

Order POLYZONIIDA

Family POLYZONIIDAE

\section{Polyzonium germanicum Brandt, 1837}

Platyiulus audouinianus Gervais, 1836

Polyzonium bosniense Verhoeff, 1898

Polyzonium albanicum Verhoeff, 1932

Polyzonium atlanticum Brolemann, 1935

Polyzonium controversarium Verhoeff, 1937

MATERIAL. 1 ○', Mavrovo, 14.VI.1996, leg. E. Stojkoska; 1 $\sigma^{7}$, near the village of Popadija, Mt Belasica, $1038 \mathrm{~m}$ a.s.1., Festuca-Fagus community, 28.V.2010; $2 \sigma^{7} \sigma^{7}$, same locality, $1100 \mathrm{~m}$ a.s.1., Festuca-Fagus community, 28.V.2010, all leg. A.C. Gjorgjievska.

Distribution in the Republic of North Macedonia: Mt Belasica (present study); Korab [Verhoeff, 1932]; Ljuboten, Šar Planina Mts [Attems, 1929; Verhoeff, 1932]; Mavrovo (present study); without precise locality [Strasser, 1971a, b; Mršić, 1993a; Mauriès et al., 1997; Makarov et al., 2004] (Fig. 2).

World distribution: Albania, Austria, Bosnia and Herzegovina, Belarus, Bulgaria, Croatia, Czech Republic, Danish mainland, Estonia, Finland, French mainland, Germany, Great Britain, Hungary, Italian mainland, Lithuania, Latvia, Republic of North Macedonia, Moldova, Montenegro, Poland, Romania, Russia (Kaliningrad Region, Central European Russia, East Central Russia, North European Russia, North- west Russia), Serbia, Slovakia, Slovenia, Sweden, and Ukraine [Kime, Enghoff, 2011].

\section{Family HIRUDISOMATIDAE}

\section{Hirudisoma hirsutum (Verhoeff, 1901)*}

Heterozonium hirsutum Verhoeff, 1901

MATERIAL. $3 \sigma^{\top} \sigma^{7}, 6$ 00 , near the village of Popadija, Mt Belasica, $847 \mathrm{~m}$ a.s.1., Quercus-Fraxinus forest, 28.V.2010; 1 ㅇ, same locality, $847 \mathrm{~m}$ a.s.1., Quercus-Fraxinus forest, 3.VII.2010, all leg. A.C. Gjorgjievska; 2 우, village of Smolare, Strumica, chestnut forest, 25.X.2010, leg. M. Komnenov.

Distribution in the Republic of North Macedonia: $\mathrm{Mt}$ Belasica (present study); Strumica (present study) (Fig. 2).

Note: This is the first record of this species in the Republic of North Macedonia, but also the first record of the genus and family for the country.

World distribution: Asiatic Turkey, Bulgaria, European Turkey (without islands in the Sea of Marmara), Republic of North Macedonia, and Greek mainland [Kime, Enghoff, 2011; present study].

\section{Order CALLIPODIDA \\ Family DORYPETALIDAE}

\section{Dorypetalum degenerans (Latzel, 1884)}

Lysiopetalum degenerans Latzel, 1884

Distribution in the Republic of North Macedonia: Kaplan, near Skopje [Attems, 1929]; Katlanovo [Mršić, 1993a]; Kosturino [Mršić, 1993a]; Strmoš, Probištip [Mršić, 1993a]; Treska [Attems, 1929]; Mt Vodno, Skopje [Mršić, 1993]; without precise locality [Strasser, 1971a; Mauriès et al., 1997; Makarov et al., 2004] (Fig. 2). 
World distribution: Bosnia and Herzegovina, Hungary, Republic of North Macedonia, Romania, and Serbia [Kime, Enghoff, 2011].

\section{Family SCHIZOPETALLIDAE}

\section{Acanthopetalum carinatum (Brandt, 1840)}

Lysiopetalum carinatum Brandt, 1840

Lysiopetalum comma Verhoeff, 1900

Lysiopetalum thessalorum Verhoeff, 1901

Lysiopetalum macedonicum Verhoeff, 1923

Lysiopetalum albanicum Verhoeff, 1932

Lysiopetalum janinense Verhoeff, 1932

Lysiopetalum lychnitis Verhoeff, 1932

Distribution in the Republic of North Macedonia: Bigor drop, Vitolište [Mršić, 1993a]; Carevec, Mt Bistra, [Mršić, 1993a]; Dojran [Mršić, 1993a]; Mt Galičica [Mršić, 1993a]; Jama Leskovec Cave, Prespa [Mršić, 1993a]; Kaldrma, Stari Dojran [Ćurčić et al., 1999a]; Maharovac Cave, near Veles [Mršić, 1993a]; Mt Korab [Verhoeff, 1932; Strasser, 1971a]; Ohrid [Attems, 1929; Verhoeff, 1932; Strasser, 1971a]; Oteševo, near Prespa [Mršić, 1993a]; Peštani, near Ohrid [Mršić, 1993a]; Skopje [Attems, 1929]; Veles [Strasser, 1971a]; Topolka, Veles [Verhoeff, 1923]; Trnica, Mt Bistra [Ćurčić et al., 1999a; Makarov et al., 2004]; Veles [Attems, 1929]; Mt Vodno [Verhoeff, 1923]; without precise locality [Attems, 1959; Mauriès et al., 1997; Ćurčić et al., 2002a, b; Makarov et al., 2004] (Fig. 2).

World distribution: Albania, Bosnia and Herzegovina, Croatia, Greek mainland, Republic of North Macedonia, and Montenegro [Kime, Enghoff, 2011].

\section{Callipodella fasciata (Latzel, 1883)**}

Lysiopetalum fasciatum Latzel, 1883

Callipodella trifasciatum (Daday, 1889)

MATERIAL. $1 \sigma^{7}$, Đuralo Cave, Makedonski Brod, 18.III.2000, leg. E. Stojkoska; 1 , Ohrid, Stari Grad, 22.VII.2009, leg. D. Stojanoviæ; 1 , near the Kolešino Waterfall, Mt Belasica, $415 \mathrm{~m}$ a.s.1., Ouercus-Carpinus forest, 24.IV.2010; 1 ․ below the vantage point near the Kolešino Waterfall, Mt Belasica, $327 \mathrm{~m}$ a.s.l., Quercus-Carpinus forest, 28.V.2010; 2 우, between the localities of Pod and Suva Češma, Mt Belasica, $587 \mathrm{~m}$ a.s.1., QuercusCarpinus forest, 28.V.2010; $1 \sigma^{7}$, near the village of Popadija, Mt Belasica, $767 \mathrm{~m}$ a.s.1., Quercus-Fraxinus forest, 28.V.2010; 1 은 same locality, $1038 \mathrm{~m}$ a.s.1., Quercus-Fraxinus forest, 3.VII.2010; $2 \sigma^{\Upsilon} \sigma^{\top}$, same locality, $1100 \mathrm{~m}$ a.s.1., Festuca-Fagus community, 3.VII.2010; 1 , near the Pisana Skala slopes, Mt Belasica, $1385 \mathrm{~m}$ a.s.1., Calamintha-Fagus community, 3.VII.2010; $1 \sigma^{7}$, near Groba, Mt Belasica, $1300 \mathrm{~m}$ a.s.1., Calamintha-Fagus community, 4.X.2010; all leg. A.C. Gjorgjievska; $1 \sigma^{7}, 4$ qo, Vodno, Skopje, $770 \mathrm{~m}$ a.s.1., 15.VI.2015; 1 9, same locality, $770 \mathrm{~m}$ a.s.1., 7.VII.2015, all leg. R. Blaževska; 1 q, village of Dolno Jelovce, Šar Planina Mts, Cornish oak forest, 6.IX.2017; 1 \%, village of Dolno Jelovce, Šar Planina Mts, oak forest, 6.IX.2017, all leg. A.C. Gjorgjievska, S. Hristovski.

Distribution in the Republic of North Macedonia: Mt Belasica (present study); Dubrovnica [Mršić, 1993a]; Golem grad, Lake Prespa [Verhoeff, 1932]; Kosturino [Mršiæ, 1993a]; Krklja, near Kriva Palanka [Mršić, 1993a]; Makedonski Brod (present study); Ohrid (present study); Šar Planina Mts (present study); Treska, near Skopje [Attems, 1929]; Mt Vodno, Skopje [Mršić, 1993a, present study]; without precise locality [Attems, 1959; Strasser, 1971a; Mauriès et al., 1997; Makarov et al., 2004] (Fig. 2).

World distribution: Albania, Bosnia and Herzegovina, Bulgaria, Greek mainland, Republic of North Macedonia, Montenegro, Romania, and Serbia [Kime, Enghoff, 2011].
Balkan endemic.

Order CHORDEUMATIDA
Family CHORDEUMATIDAE

\section{Melogona broelemanni (Verhoeff, 1897)}

Microchordeuma broelemanni Verhoeff, 1897

Microchordeuma illyricum Verhoeff, 1932

Microchordeuma gebhardti Loksa, 1962

MATERIAL. 1 \%, Gostivar, date and leg.: no data; $1 \sigma^{r}, 1$ ㅇ same locality, date and leg.: no data; $1 \sigma^{7}, 1$, village of Rašèe, near Skopje, 1.V.1996; 1 \%, Monastery of St. Jovan Bigorski, Mt Bistra, 13.IV.2006; $1 \sigma^{7}$, Smrdliva Voda, Mt Kožuf, 700 m a.s.1., 25.IV.2010, all leg. I. Karaman; $1 \sigma^{7}$, near the Kolešino Waterfal, Mt Belasica, $415 \mathrm{~m}$ a.s.1., Quercus-Carpinus forest, 24.IV.2010; 1 $\mathrm{O}^{7}, 2$ 우, near the village of Popadija, Mt Belasica, $1100 \mathrm{~m}$ a.s.1., Festuca-Fagus community, 28.V.2010; $1 \sigma^{7}$, same locality, 1200 $\mathrm{m}$ a.s.1., 28.V.2010; $5 \sigma^{7} \sigma^{7}, 1$, near the Pisana Skala slopes, Mt Belasica, $1385 \mathrm{~m}$ a.s.1., 28.V.2010, all leg. A.C. Gjorgjievska.

Distribution in the Republic of North Macedonia: Mt Belasica (present study); Mt Bistra (present study); Mt Kožuf (present study); Ljuboten, Šar Planina Mts [Attems, 1929]; Nerezi, near Skopje [Attems, 1929]; Gostivar (present study); Rašče, near Skopje (present study); village of Sasa [Mršić, 1993a]; without precise locality [Strasser, 1971a; Mauriès et al., 1997; Makarov et al., 2004] (Fig. 2).

World distribution: Albania, Austria, Bosnia and Herzegovina, Bulgaria, Croatia, Czech Republic, Greek mainland, Hungary, Republic of North Macedonia, Montenegro, Romania, Serbia, and Slovenia [Kime, Enghoff, 2013].

\section{Family NEOATRACTOSOMATIDAE}

\section{Paeonisoma faucium Verhoeff, 1932**}

Distribution in the Republic of North Macedonia: Kakalo, Mt Jablanica [Verhoeff, 1932; Strasser, 1971a; Mršić, 1993a]; without precise locality [Mauriès et al., 1997; Ćurčić et al., 2002a; Makarov et al., 2004] (Fig. 2).

Note: So far, one adult female and juveniles are the only known specimens of this species. According to Mauriès [2003] it is possible that Paeonisoma and Schizmohetera are related or that Paeonisoma is a synonym of Schizmohetera, since Paeonisoma faucium specimens were found relatively near the type locality of Schizmohetera sketi and the vulvae of these two species are similar. Until males are found, precise answers cannot be given.

Mauriès et al. [1997] included this species in the checklist of millipedes of Albania and adjacent lands, based on findings by Ceuca [1992] and their own findings, but they did not provide with any information about the record of $P$. faucium in Albania.

North Macedonian endemic.

\section{Schizmohetera curcici Makarov, 2001**}

Distribution in the Republic of North Macedonia: Kalina Dupka Cave, village of Lazaropole, Mt Bistra [Makarov, 2001; Makarov et al., 2004] (Fig. 2).

North Macedonian endemic.

\section{Schizmohetera sketi Mršić, 1987**}

Distribution in Republic of North Macedonia: Alilica Cave, Mt Bistra [Mršić, 1987, 1993a]; without precise locality [Ćurčić et al., 2002a, b; Makarov et al., 2004] (Fig. 2). 
North Macedonian endemic.

Order JULIDA

Family BLANIULIDAE

\section{Nopoiulus kochii (Gervais, 1847)}

Iulus kochii Gervais, 1847

Nopoiulus pulchellus auct., not C.L. Koch (1838)

Nopoiulus minutus auct., not Brandt (1841)

Blaniulus venustus Meinert, 1868

Nemasoma minutum Bollman, 1888

Blaniulus armatus Nemeè, 1895

Gomphiocephalus gracilis (Verhoeff, 1943)

Nopoiulus breuili Brolemann, 1921

Nopoiulus subtilis Brolemann, 1923

Blaniulus atticus Verhoeff, 1925

Distribution in the Republic of North Macedonia: Without precise locality [Mršić, 1993a; Mauriès et al., 1997; Makarov et al., 2004].

World distribution: Cosmopolitan [Kime, Enghoff, 2017].

Family JULIDAE

\section{Acanthoiulus fuscipes (C. L. Koch, 1847)}

Julus fuscipes C.L. Koch, 1847

Julus idriensis C.L. Koch, 1847

Julus dalmaticus C.L. Koch, 1847

Iulus leuconotus Latzel, 1884

Iulus subcrassus Latzel, 1884

Pachyiulus bosniensis Verhoeff, 1895

Pachyiulus krohnii Verhoeff, 1898

Pachyiulus antivagus Verhoeff, 1899

Pachyiulus plasensis Verhoeff, 1910

Pachyiulus simplex Verhoeff, 1910

Pachyiulus fuscipes auct.

Distribution in the Republic of North Macedonia: Beli near Kočani [Mršić, 1993a]; Mt Bistra [Mršić, 1993a]; Istibanja [Mršić, 1993a]; Katlanovo [Mršić, 1993a]; Kosturino [Mršić, 1993a]; Ljuboten, Šar Planina Mts [Attems, 1929]; Matka, Dogadžica [Mršiæ, 1993a]; Babunište, Struško [Mršić, 1993a]; village of Sasa [Mršić, 1993a]; Staro Nagoričane [Mršić, 1993a]; Stobi [Mršić, 1993a]; Struško [Mršić, 1993a]; river Treska, near Skopje [Attems, 1929]; Mt Vodno, Skopje [Mršić, 1993a]; without precise locality [Strasser, 1971a; Makarov et al., 2004] (Fig. 3).

World distribution: Albania, Bosnia and Herzegovina, Croatia, Greece (Peloponnese); Italian mainland, Republic of North Macedonia, Montenegro, Serbia, and Slovenia [Kime, Enghoff, 2017].

\section{Brachyiulus lusitanus Verhoeff, 1898}

Brachyiulus corcyraeus Verhoeff, 1900

Microbrachyiulus calcivagus Verhoeff, 1910

Distribution in the Republic of North Macedonia: Without precise locality [Strasser, 1971a; Mršić, 1993a; Mauriès et al., 1997; Makarov et al., 2004].

World distribution: Austria, Azores Islands, Balearic Islands, Bulgaria, Canary Islands, Corsica, Croatia, Czech Republic, Germany, Great Britain, Greek mainland including Ionian Islands, Italian mainland, Republic of North Macedonia, Madeira Islands, Monaco, Portuguese mainland, Sardinia, Spanish mainland, and Switzerland, as well as Algeria, Egypt, Iran, Australia, North America, and possibly Hungary [Kime, Enghoff, 2017].

\section{Brachyiulus bagnalli (Brolemann, 1924)}

Microbrachyiulus bagnalli Brolemann, 1924

Brachyiulus latzeli Attems, 1949

Distribution in the Republic of North Macedonia: Skopje [Mršić, 1993a] (Fig. 3).

World distribution: Austria, Bulgaria, Czech Republic, Hungary, Republic of North Macedonia, Poland, Romania, Serbia, Slovakia, and Slovenia [Kime, Enghoff, 2017].

\section{Chromatoiulus hamuligerus (Verhoeff,} 1932)**

Pachybrachyiulus hamuligerus Verhoeff, 1932

Leptomastigoiulus hamuligerus Mršić, 1993

Distribution in the Republic of North Macedonia: Mt Bistra, Mavrovo [Mršić, 1993a]; Jablanica, Mt Kakalo [Verhoeff, 1932]; Mt Korab [Verhoeff, 1932; Strasser, 1971a]; without precise locality [Mauriès et al., 1997; Curčić et al., 2002a; Makarov et al., 2004] (Fig. 3).

North Macedonian endemic.

\section{Chromatoiulus podabrus (Latzel, 1884)}

Iulus podabrus Latzel, 1884

Julus bosniensis Latzel, 1888

Brachyiulus krohnii Verhoeff, 1898

Pachybrachyiulus podabrus auct.

MATERIAL. $1 \sigma^{7}$, Jelak, Šar Planina Mts, date and leg.: no data; $1 \Im^{\top}, 1$ ㅇ, village of Raduša, Mt Žeden, 15.IV.1994, leg.: no data; 1 , St. Ilija Monastery, Skopska Crna Gora, 13.X.2000, legs. S. Stanković, E. Stojkoska.

Distribution in the Republic of North Macedonia: Ljuboten, Šar Planina Mts [Attems, 1929; present study]; Skopska Crna Gora (present study); Treska [Attems, 1929]; Mt Žeden (present study); without precise locality [Strasser, 1971a; Mršić, 1993a; Mauriès et al., 1997; Makarov et al., 2004] (Fig. 3).

World distribution: Albania, Bosnia and Herzegovina, Croatia, Republic of North Macedonia, Greek mainland including Ionian Islands, Italian mainland, Montenegro, and Serbia [Kime, Enghoff, 2017].

\section{Cylindroiulus arborum Verhoeff, 1928}

Allajulus arborum (Verhoeff, 1928)

Distribution in the Republic of North Macedonia: Stenje, Lake Prespa [Mršić, 1993a; Makarov et al., 2004] (Fig. 3A).

World distribution: Austria, Belgium, Bulgaria, Czech Republic, Germany, Greek mainland, Hungary, Lithuania, Republic of North Macedonia, Poland, Romania, Slovakia, and Ukraine, with a record from the Northern Caucasus region, Russia [Kime, Enghoff, 2017].

\section{Cylindroiulus boleti (C.L. Koch, 1847)}

Iulus boleti C.L. Koch, 1847

Diploiulus boleti auct.

MATERIAL. $1 \sigma^{7}, 2$ 오, village of Smojmirovo, Berovo, 924$932 \mathrm{~m}$ a.s.l., ecotope between pine forest and open area dominated by Juniperus communis and Pinus silvestris, IV.2014, leg. D. Matevski.

Distribution in the Republic of North Macedonia: Berovo (present study), Crn Kamen, Mt Korab [Verhoeff, 1932]; Dragomance, near Kumanovo [Mršić, 1993a]; Katlanovo [Mršić, 1993a]; Ljuboten, Šar Planina Mts [Attems, 1929]; 


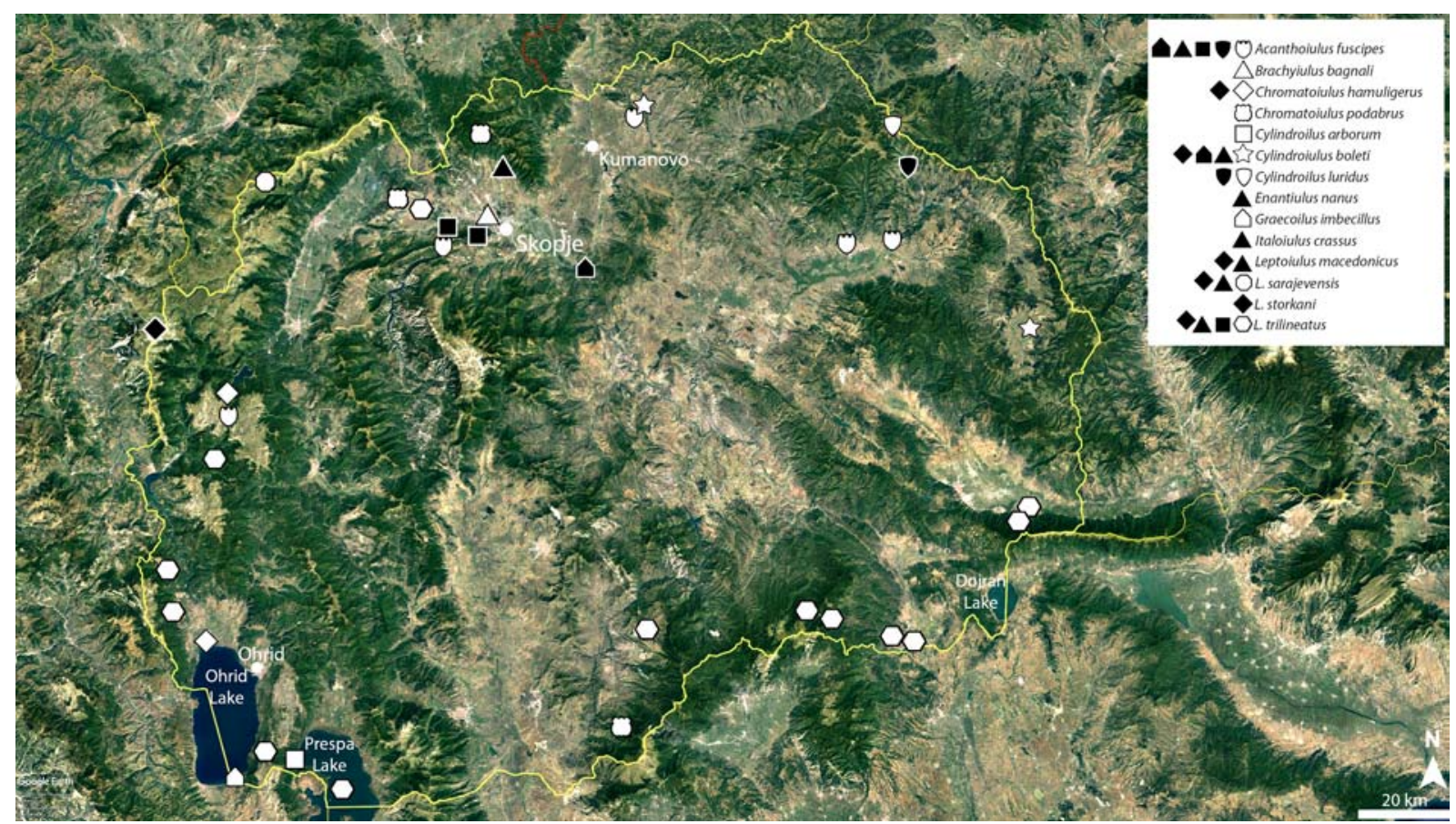

Fig. 3. Distribution of representatives of the order Julida (family Julidae) in North Macedonia.

Рис. 3. Распространение представителей отряда Julida (семейство Julidae) в Северной Македонии.

without precise locality [Strasser, 1971a; Mauriès et al., 1997; Makarov et al., 2004] (Fig. 3).

World distribution: Albania, Austria, Bosnia and Herzegovina, Bulgaria, Croatia, Czech Republic, Germany, Hungary, Italian mainland, Republic of North Macedonia, Moldova, Montenegro, Romania, Serbia, Slovakia, Slovenia, and Ukraine [Kime, Enghoff, 2017].

\section{Cylindroiulus luridus (C.L. Koch, 1847)}

Julus luridus C.L. Koch, 1847

MATERIAL. $2 \sigma^{7} \sigma^{7}$, Gumnove Livade, Mt Osogovo, 31.V. 2000, leg. E. Stojkoska.

Distribution in the Republic of North Macedonia: Mt Osogovo (present study); village of Sasa [Mršić, 1993a] (Fig. 3).

World distribution: Austria, Bosnia and Herzegovina, Bulgaria, Croatia, Czech Republic, Germany, Hungary, Italian mainland, Republic of North Macedonia, Romania, Serbia, Slovakia, and Slovenia [Kime, Enghoff, 2017].

\section{Enantiulus nanus (Latzel, 1884)}

Allajulus albicornis C.L. Koch, 1847

Iulus nanus Latzel, 1884

Leptophyllum nanum auct.

Distribution in the Republic of North Macedonia: Ljuboten, Šar Planina Mts [Attems, 1929; Makarov et al., 2004] (Fig. 3).

World distribution: Albania, Bosnia and Herzegovina, Belgium, Bulgaria, Croatia, Czech Republic, Danish mainland, French mainland, Germany, Hungary, Italian mainland, Lithuania, Luxembourg, Republic of North Macedonia, Montenegro, the Netherlands, Poland, Romania, Serbia, Slovakia, Slovenia, Sweden, Switzerland, and Ukraine [Kime, Enghoff, 2017].

\section{Graecoiulus imbecillus (Attems, 1935)**}

Chromatoiulus imbecillus Attems, 1935

Megaphyllum imbecillum Mauriès et al., 1997

Distribution in the Republic of North Macedonia: St. Naum Monastery, Lake Ohrid [Lazányi et al., 2012] (Fig. 3). World distribution: Albania, Greece, Republic of North Macedonia [Lazányi et al., 2012; Vagalinski, Lazányi, 2018]. Balkan endemic.

\section{Italoiulus crassus (Attems, 1929)**}

Chromatoiulus crassus Attems, 1929

Megaphyllum crassum Mršić, 1993

Distribution in the Republic of North Macedonia: Ljuboten, Šar Planina Mts [Attems, 1929; Strasser, 1971a]; without precise locality [Mršić, 1993a; Mauriès et al., 1997; Ćurčić et al., 2002a; Makarov et al., 2004] (Fig. 3).

World distribution: Republic of North Macedonia, Serbia [Lazányi et al., 2012; Vagalinski, Lazányi, 2018].

Balkan endemic.

\section{Leptoiulus macedonicus (Attems, 1927)**}

Microiulus macedonicus Attems, 1927

Xestoiulus macedonicus auct.

Distribution in the Republic of North Macedonia: Duboka Rečka, Mt Korab [Verhoeff, 1932]; Mt Korab [Attems, 1927; Strasser, 1976]; Ljuboten, Šar Planina Mts [Attems, 1927, 1929; Verhoeff, 1932; Strasser, 1971a, 1976; Mršić, 1988a]; without precise locality [Mršić, 1993a; Mauriès et al., 1997; Ćurčić et al., 2002a; Makarov et al., 2004] (Fig. 3).

World distribution: Albania, Greek mainland, Republic of North Macedonia, and Serbia [Kime, Enghoff, 2017].

Balkan endemic. 


\section{Leptoiulus sarajevensis Verhoeff, 1898**}

Julus sarajevensis Verhoeff, 1898

Macedoiulus storkani Verhoeff, 1932

MATERIAL. 1 \%, 1 ㅇ, Lake Brezno, Šar Planina Mts, 6.IX 2017, leg. A.C. Gjorgjievska, S. Hristovski.

Distribution in the Republic of North Macedonia: Lake Brezno, Šar Planina Mts (present study), Čošelija, Mt Korab [Verhoeff, 1932]; Ljuboten, Šar Planina Mts [Attems, 1929]; without precise locality [Strasser, 1971a; Mršić, 1988a, 1993a; Mauriès et al., 1997; Makarov et al., 2004] (Fig. 3).

World distribution: Bosnia and Herzegovina, Bulgaria, Croatia, Republic of North Macedonia, Montenegro, and Serbia [Kime, Enghoff, 2017].

Balkan endemic.

\section{Leptoiulus storkani Verhoeff, 1932**}

Microiulus storkani auct.

Xestoiulus storkani auct.

Leptoiulus jaroslavi Mauriès et al., 1997

Distribution in the Republic of North Macedonia: Coselija, Mt Korab [Verhoeff, 1932]; Duboka Rečka, Mt Korab [Verhoeff, 1932; Strasser, 1971a]; Mt Korab [Strasser, 1976]; without precise locality [Strasser, 1976; Mršić, 1993a; Mauriès et al., 1997; Ćurčić et al., 2002a; Makarov et al., 2004] (Fig. 3).

Note: Kime \& Enghoff [2017] question the presence of this species in Albania, Croatia, and Greece. Mauriès et al. [1997] remark that Strasser [1976] included L. storkani in the millipede fauna of Albania, without providing information about the records.

World distribution: Republic of North Macedonia and possibly Albania, Croatia, and Greece [Kime, Enghoff, 2017].

Balkan endemic.

\section{Leptoiulus trilineatus (C.L. Koch, 1847)}

Julus trilineatus C.L. Koch, 1847

Julus silvivagus Verhoeff, 1898

Julus plasensis Verhoeff, 1908

Leptoiulus luganensis Verhoeff, 1913

Leptoiulus bureschi Verhoeff, 1928

MATERIAL. $1 \Im^{7}$, Gostivar, date and leg.: no data; 1 , $2 \bigcirc^{\top} \sigma^{\top}$, village of Rašče, near Skopje, 1.V.1996, leg. I. Karaman; $1 \sigma^{7}$, near the village of Popadija, Mt Belasica, $1200 \mathrm{~m}$ a.s.1., CalaminthaFagus community 24.IV.2010; 1 乃", same locality, $1100 \mathrm{~m}$ a.s.1., Festuca-Fagus community, 28.V.2010; $3 \sigma^{7} \sigma^{7}$, same locality, 1200 $\mathrm{m}$ a.s.1., 28.V.2010; $1 \sigma^{7}$, below the vantage point near the Kolešino Waterfall, Mt Belasica, 327 m a.s.1., Quercus-Carpinus forest, 28.V.2010, all leg. A.C. Gjorgjievska; 1 ア, Mt Vodno, Skopje, $770 \mathrm{~m}$ a.s.1., Mediterranean pine forest, 15.VI.2010, leg. R. Blaževska; 1 ア', Gorska Buka, Mt Kožuf, 1505 m a.s.l., CalaminthaFagus community, 14.VI.2016; 1 \%,. Mt Kožuf, 679 m a.s.1. Festuca-Fagus community, 14.VI.2016; 1 ○', Mt Kožuf, 87 m a.s.1., Quercus-Carpinus forest, 16.VI.2016, all leg. D. Matevski; $1 \sigma^{7}$, St. Spas, Mt Jablanica, $1267 \mathrm{~m}$ a.s.1., chestnut forest, 15.IX. 2017; 1 フ , Vevčanski Izvori, Mt Jablanica, 1300 m a.s.1., 15.X. 2017, all leg. A. Taseska.

Distribution in the Republic of North Macedonia: Mt Belasica (present study); Bigor drop, Vitolište [Mršić, 1993a]; Gabrovo reservoir, Gevgelija [Mršić, 1993a]; Mt Galièica [Mršić, 1993a]; Mt Jablanica [Verhoeff, 1932; present study]; Mt Korab [Verhoeff, 1932]; Mt Kožuf (present study); Lazaropole, Mt Bistra [Ćurčić et al., 1999a]; Ljuboten, Šar Planina Mts [Verhoeff, 1932]; Lake Prespa [Verhoeff, 1932]; Stenje, Lake Prespa [Mršić, 1993a]; Treska, near Skopje [Attems, 1929; Mršić, 1993a]; Mt Vodno, Skopje (present study); without precise locality [Makarov et al., 2004] (Fig. 3).

World distribution: Albania, Austria, Bosnia and Herzegovina, Bulgaria, Croatia, European Turkey (without islands in the Sea of Marmara), Greek mainland including Ionian Islands, Italian mainland, Montenegro, Romania, Serbia, Slovenia, and possibly Switzerland [Kime, Enghoff, 2017].

\section{Megaphyllum austriacum (Latzel, 1884)}

Iulus austriacus Latzel, 1884

Brachyiulus austriacus auct.

Chromatoiulus austriacus auct.

Brachyiulus dahli Verhoeff, 1901

Chromatoiulus dahli auct.

Distribution in the Republic of North Macedonia: along the Treska River, Skopje [Ćurčić et al., 1999a; Makarov et al., 2004] (Fig. 4).

World distribution: Bosnia and Herzegovina, Croatia, Italian mainland, Republic of North Macedonia, Montenegro, Serbia, and Slovenia [Kime, Enghoff, 2017].

\section{Megaphyllum bosniense (Verhoeff, 1897)}

Brachyiulus bosniensis Verhoeff, 1897

Chromatoiulus bosniensis auct.

Chromatoiulus cotinophilus Loksa, 1962

MATERIAL. 1 +, Gostivar, date and leg.: no data; $1 \sigma^{7}$, Kratovo, 3.VIII.2009; 3 o $^{7}$, near Groba, Mt Belasica, 1300 m a.s.1., Calamintha-Fagus community, 24.IV.2010; $1 \sigma^{7}$, same locality, $1300 \mathrm{~m}$ a.s.1., Calamintha-Fagus community, 4.IX.2010; 1 \%", near the village of Popadija, Mt Belasica, $1100 \mathrm{~m}$ a.s.1., FestucaFagus community, 28.V.2010; $2 \sigma^{\top} \sigma^{\top}, 2$ ơ, same locality, $1200 \mathrm{~m}$ a.s.1., Calamintha-Fagus community, 28.V.2010; $3 \sigma^{7} \sigma^{7}$, near the Pisana Skala slopes, Mt Belasica, $1385 \mathrm{~m}$ a.s.1., Calamintha-Fagus community, 28.V.2010; $2 \sigma^{\top} \sigma^{\top}, 5$ 우, same locality, $1442 \mathrm{~m}$ a.s.1., cleared area, 28.V.2010; 1 \%, same locality, $1385 \mathrm{~m}$ a.s.1., Calamintha-Fagus community, 3.VII.2010, all leg. A.C. Gjorgjievska; 1 + Mt Kožuf, 679 m a.s.1., Festuca-Fagus community, 16.VI.2016; 1 ऊ', Mt Kožuf, 896 m a.s.1., Festuca-Fagus community, 19.VII.2016, all leg. D. Matevski.

Distribution in the Republic of North Macedonia: Mt Belasica (present study); Bregalnica River [Mršić, 1993a]; Carevec, Mt Bistra [Mršić, 1993a]; Ceripašina peak, Šar Planina Mts [Mršić, 1993a]; Dragomance, near Kumanovo [Mršić, 1993a]; Mt Galièica [Mršić, 1993a]; Mt Jablanica [Verhoeff, 1932]; Katlanovo [Mršić, 1993a]; Mt Korab [Verhoeff, 1932]; Mt Kožuf (present study); valley of the Bregalnica River [Mršić, 1993a]; Kriva Reka, Kriva Palanka [Mršić, 1993a]; Ljuboten, Šar Planina Mts [Attems, 1929]; Nerezi, near Skopje [Attems, 1929]; near Lake Prespa [Verhoeff, 1932]; village of Sasa [Mršić, 1993a]; Skopje [Attems, 1929]; Treska, near Skopje [Attems, 1929]; without precise locality [Strasser, 1971a; Mauriès et al., 1997; Makarov et al., 2004] (Fig. 4).

World distribution: Albania, Austria, Bosnia and Herzegovina, Bulgaria, Croatia, Greek mainland, Hungary, Italian mainland, Republic of North Macedonia, Montenegro, Romania, Serbia, and Slovenia [Kime, Enghoff, 2017].

\section{Megaphyllum dentatum (Verhoeff, 1898)**}

Brachyiulus dentatus Verhoeff, 1898

Chromatoiulus dentatus auct.

Distribution in the Republic of North Macedonia: Ceripašina peak, Šar Planina Mts [Strasser, 1971a]; Mt Jablani- 


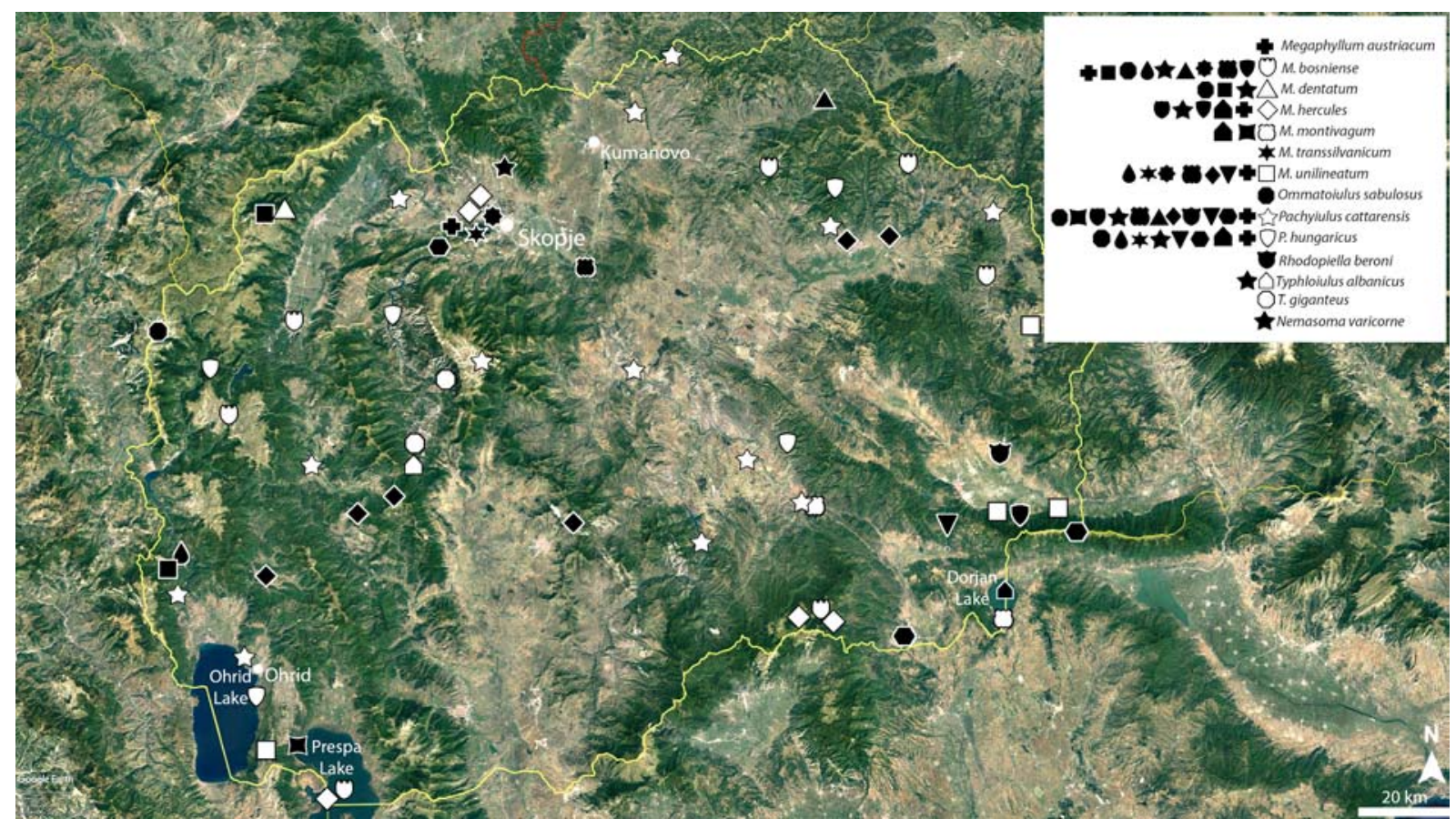

Fig. 4. Distribution of representatives of the order Julida (families Julidae and Nemasomatidae) in North Macedonia.

Рис. 4. Распространение представителей отряда Julida (семейства Julidae и Nemasomatidae) в Северной Македонии.

ca [Verhoeff, 1932]; Mt Korab [Verhoeff, 1932]; Ljuboten, Šar Planina Mts [Attems, 1929; Verhoeff, 1932; Makarov et al., 2004]; Popova Šapka, Šar Planina Mts [Mršić, 1993a] (Fig. 4).

World distribution: Albania, Bosnia and Herzegovina, Bulgaria, Republic of North Macedonia, Montenegro, and Serbia [Kime, Enghoff, 2017].

Balkan endemic.

\section{Megaphyllum hercules (Verhoeff, 1900)}

Brachyiulus hercules Verhoeff, 1900

Brachyiulus tauricus Attems, 1907

Chromatoiulus hercules auct.

MATERIAL. 1 , , village of Kolešino, Mt Belasica, 20.X.2000, legs. E. Stojkoska, S. Stankoviæ; 1 \%, Mt Kožuf, $550 \mathrm{~m}$ a.s.1., 15.VI.2010, leg.: no data; 2 우, near the Pisana Skala slopes, Mt Belasica, $1442 \mathrm{~m}$ a.s.1., cleared area, 3.VII.2010, leg. A.C. Gjorgjievska; 1 , municipality of Sonje, Skopje, $478 \mathrm{~m}$ a.s.1., thermoMediterranean shrubby formation, 10.VI.2015; 1 , 1 juv., same locality, $478 \mathrm{~m}$ a.s.l., thermo-Mediterranean shrubby formation, 5.VII.2015; 1 , , village of Vizbegovo, Skopje, $146 \mathrm{~m}$ a.s.1., Mediterranean xerothermal grassy vegetation, 10.VI.2015, all leg. R. Blaževska; 1 q. Mt Kožuf, 1672 m a.s.1., Fagus-Pinus forest, 15.VI.2016, leg. D. Matevski.

Distribution in the Republic of North Macedonia: Golem Grad near Prespa [Verhoeff, 1932]; Karadojranski Potok, Lake Dojran [Ćurčić et al., 1999a]; Ljuboten, Šar Planina Mts [Verhoeff, 1932]; Treska [Attems, 1929]; Mt Vodno, Skopje [Verhoeff, 1932]; without precise locality [Strasser, 1971a; Mršić, 1993a; Mauriès et al., 1997; Makarov et al., 2004] (Fig. 4).

World distribution: Albania, Bulgaria, Greek mainland including Ionian Islands, Republic of North Macedonia, and Ukraine [Kime, Enghoff, 2017].

\section{Megaphyllum montivagum (Verhoeff, 1901)**}

Brachyiulus montivagus Verhoeff, 1901

Brachyiulus latesquamosus Attems, 1903

Chromatoiulus montivagus auct.

Chromatoiulus latesquamosus auct.

Chromatoiulus macedonicus Strasser, 1976

Megaphyllum latesquamosus auct.

Megaphyllum macedonicum auct.

MATERIAL. $5 \sigma^{7} \sigma^{7}, 9$ 9 , Dojran, Mrdaja, date and leg.: no data; 4 O $^{7}$, Lake Dojran, 10.XI.1999, leg. E. Stojkoska, S. Stanković.

Distribution in the Republic of North Macedonia: Demir Kapija, Vardar River [Lazányi et al., 2012]; Lake Dojran (present study); Lake Prespa and lakeshore south of Oteševo [Lazányi et al., 2012] (Fig. 4).

World distribution: Greece, Republic of North Macedonia, and Serbia [Kime, Enghoff, 2017].

Balkan endemic.

\section{Megaphyllum transsylvanicum (Verhoeff, 1897)}

Brachyiulus transsylvanicus Verhoeff, 1897

Brachyiulus croaticus Verhoeff, 1929

Chromatoiulus transdanubicus Loksa, 1962

Chromatoiulus transsylvanicus auct.

Distribution in the Republic of North Macedonia: Mt Vodno, near Skopje [Mršić, 1993a; Makarov et al., 2004] (Fig. 4).

World distribution: Bosnia and Herzegovina, Bulgaria, Croatia, European Turkey (without islands in the Sea of Marmara), Greek mainland, Republic of North Macedonia, Moldova, Romania, Russia (South European), Serbia, and Ukraine [Kime, Enghoff, 2017]. 


\section{Megaphyllum unilineatum (C.L. Koch, 1838)}

Julus unilineatus C.L. Koch, 1838

Julus gilvolineatus L. Koch, 1881

Julus balearicus L. Koch, 1881

Julus frivaldszkyi Daday, 1889

Brachyiulus balticus Verhoeff, 1907

Brachyiulus degenerans Verhoeff, 1937

Brachyiulus genuinus Verhoeff, 1907

Chromatoiulus unilineatus auct.

MATERIAL. $2 \Im^{\top} \sigma^{\top}$, village of Smojmirovo, Berovo, Meadow, 923-932 m a.s.1., open area (grassland) dominated by Achillea millefollium, Ononis spinosa, Galium verum, and Hypericum purpurea, VII.2014, leg. D. Matevski.

Distribution in the Republic of North Macedonia: Bansko [Mršić, 1993a]; Belgište-Blato [Mršić, 1993a]; Belčišta (Ohrid) [Mršić, 1993a]; Beli, near Koèani [Mršić, 1993a]; Berovo (present study); Mt Galičica, near Česma [Mršić, 1993a]; Istibanja [Mršić, 1993a]; Katlanovo [Mršić, 1993a]; Kosturino [Mršić, 1993a]; Nerezi, near Skopje [Attems, 1929]; Oreovec [Mršić, 1993a]; Ramnište, Cer [Mršić, 1993a]; Skopje [Mršić, 1993a]; Smolari, Mt Belasica [Mršić, 1993a]; Treska, near Skopje [Attems, 1929]; Mt Vodno [Mršić, 1993a] (Fig. 4).

World distribution: Albania, Austria, Balearic Islands, Bosnia and Herzegovina, Bulgaria, Croatia, Czech Republic, Germany, Greek mainland, Hungary, Italian mainland, Republic of North Macedonia, Montenegro, Poland, Romania, Serbia, Slovakia, and Slovenia [Kime, Enghoff, 2017].

\section{Ommatoiulus sabulosus (Linnaeus, 1758)}

Julus sabulosus Linnaeus, 1758

Iulus aimatopodus Risso, 1826

Julus bilineatus C.L. Koch, 1838

Julus rubripes C.L. Koch, 1847

Archiulus sabulosus auct.

Palaioiulus sabulosus auct.

Schizophyllum sabulosum auct.

Archiulus irregularis Attems, 1927

Ommatoiulus irregularis auct.

Distribution in the Republic of North Macedonia: Crn Kamen, Mt Korab [Verhoeff, 1932]; without precise locality [Strasser, 1971a; Mršić, 1993a; Makarov et al., 2004] (Fig. 4).

World distribution: Albania, Austria, Belgium, Bosnia and Herzegovina, Bulgaria, Belarus, Channel Islands, Croatia, Czech Republic, Danish mainland, Finland, French mainland, Germany, Great Britain, Northern Ireland, Hungary, Ireland, Italian mainland, Lithuania, Luxembourg, Latvia, Republic of North Macedonia, Monaco, Norwegian mainland and inshore islands, Poland, Romania, Russia (Kaliningrad Region, as well as Central European, East Central, and North European Russia), Serbia, Slovakia, Slovenia, Spanish mainland, Sweden, Switzerland, the Netherlands, and Ukraine [Kime, Enghoff, 2017].

\section{Pachyiulus cattarensis (Latzel, 1884)}

Iulus cattarensis Latzel, 1884

Pachyiulus longelobatus Attems, 1906

Pachyiulus venetus Verhoeff, 1926

Pachyiulus flavipes catterensis auct.

Distribution in the Republic of North Macedonia: Babuna [Mršić, 1993a]; Belčišta (Ohrid) [Mršić, 1993a]; Belasica [Mršić, 1993a]; Belgište-Blato [Mršić, 1993a]; Beli, Kočani [Mršić, 1993a]; Crn Kamen, Mt Korab [Verhoeff,
1932]; Demir Kapija [Mršić, 1993a]; Gabrovo accumulation, Gevgelija [Mršić, 1993a]; Golem Grad, Lake Prespa [Verhoeff, 1932]; near Hamzali, Mt Ogražden [Mršić, 1993a]; Istibanja [Mršić, 1993a]; Jakupica [Mršić, 1993a]; Katlanovo [Mršić, 1993a]; Kičevsko [Mršić, 1993a]; Kosturino [Mršić, 1993a]; Kozjak, Majdan [Mršić, 1993a]; Kriva Palanka [Mršić, 1993a]; Matka [Mršić, 1993a]; Nivièani near Kočani [Mršić, 1993a]; Ohrid [Verhoeff, 1932]; Oskomci, Kičevo [Mršić, 1993a]; Oreovec (Makedonski Brod) [Mršić, 1993a]; Oteševo [Mršić, 1993a]; Babunište, Struško [Mršić, 1993a]; Radoviš-Negotino, Mt Konečka [Mršić, 1993a]; Raduša, Mt Žeden [Ćurčić et al., 1999a]; Ramnište, CerMakedonski Brod [Mršić, 1993a]; Staro Nagoričane [Mršić, 1993a]; Lake Tikveš [Mršić, 1993a]; Treska, Skopje [Attems, 1929]; Mt Vodno, Skopje [Mršić, 1993a]; without precise locality [Strasser, 1971a; Mauriès et al., 1997; Makarov et al., 2004]; Zvegor, Delčevo [Mršić, 1993a] (Fig. 4).

World distribution: Albania, Asiatic Turkey, Bosnia and Herzegovina, Bulgaria, Croatia, European Turkey (without islands in the Sea of Marmara), Greek mainland including Ionian Islands, Italian mainland, Republic of North Macedonia, Montenegro, and Serbia [Kime, Enghoff, 2017].

\section{Pachyiulus hungaricus (Karsch, 1881)}

Julus hungaricus Karsch, 1881

Pachyiulus gracilis Verhoeff, 1928

MATERIAL. 1 q, St. Nikola Monastery, Matka, 30.V.2000, leg. E. Stojkoska; 1 O', Kočansko, Ponikva, 18.VII.2001, leg. E. Stojkoska, S. Stanković.

Distribution in the Republic of North Macedonia: Mt Belasica [Mršić, 1993a]; Crn Kamen, Mt Korab [Verhoeff, 1932]; Karadojranski Potok, Lake Dojran [Ćurčić et al., 1999a]; Kočansko, Ponikva (present study); Konjsko, Lake Ohrid [Verhoeff, 1932]; Kosturino [Mršić, 1993a]; Kozjak, Treska [Ćurčić et al., 1999a]; Mt Konečka [Mršić, 1993a]; Mt Kožuf [Mršić, 1993a]; Ljuboten, Šar Planina Mts [Attems, 1929]; Matka [Mršić, 1993a; present study]; Nerezi, near Skopje [Attems, 1929]; Treska, Skopje [Attems, 1929]; Trnica, Mt Korab [Verhoeff, 1932]; Mt Vodno, Skopje [Mršić, 1993a]; without precise locality [Strasser, 1971a; Mauriès et al., 1997; Makarov et al., 2004] (Fig. 4).

World distribution: Albania, Bosnia and Herzegovina, Bulgaria, Croatia, Greek mainland, Republic of North Macedonia, Montenegro, Romania, and Serbia [Kime, Enghoff, 2017].

\section{Rhodopiella beroni (Strasser, 1966)**}

Apfelbeckiella beroni Strasser, 1966

Mesoiulus bulgaricus Gulička, 1967

Apfelbeckiella saetigera Strasser, 1973

Distribution in the Republic of North Macedonia: Hamzali, Mt Ogražden [Mršić, 1993a] (Fig. 4).

World distribution: Bulgaria, Greek mainland, and Republic of North Macedonia [Kime, Enghoff, 2017].

Balkan endemic.

\section{Typhloiulus albanicus Attems, 1929**}

Distribution in the Republic of North Macedonia: Ljuboten, Šar Planina Mts [Attems, 1929]; Ovčarska Cave, Makedonski Brod [Ćurčić et al., 2002b; Makarov et al., 2004]; Skopje, Matka, Propast Srt (pot hole) [Vagalinski et al., 2015]; without precise locality [Strasser, 1962; Mršić, 1993; Mauriès et al., 1997; Ćurčić et al., 2002a] (Fig. 4). 


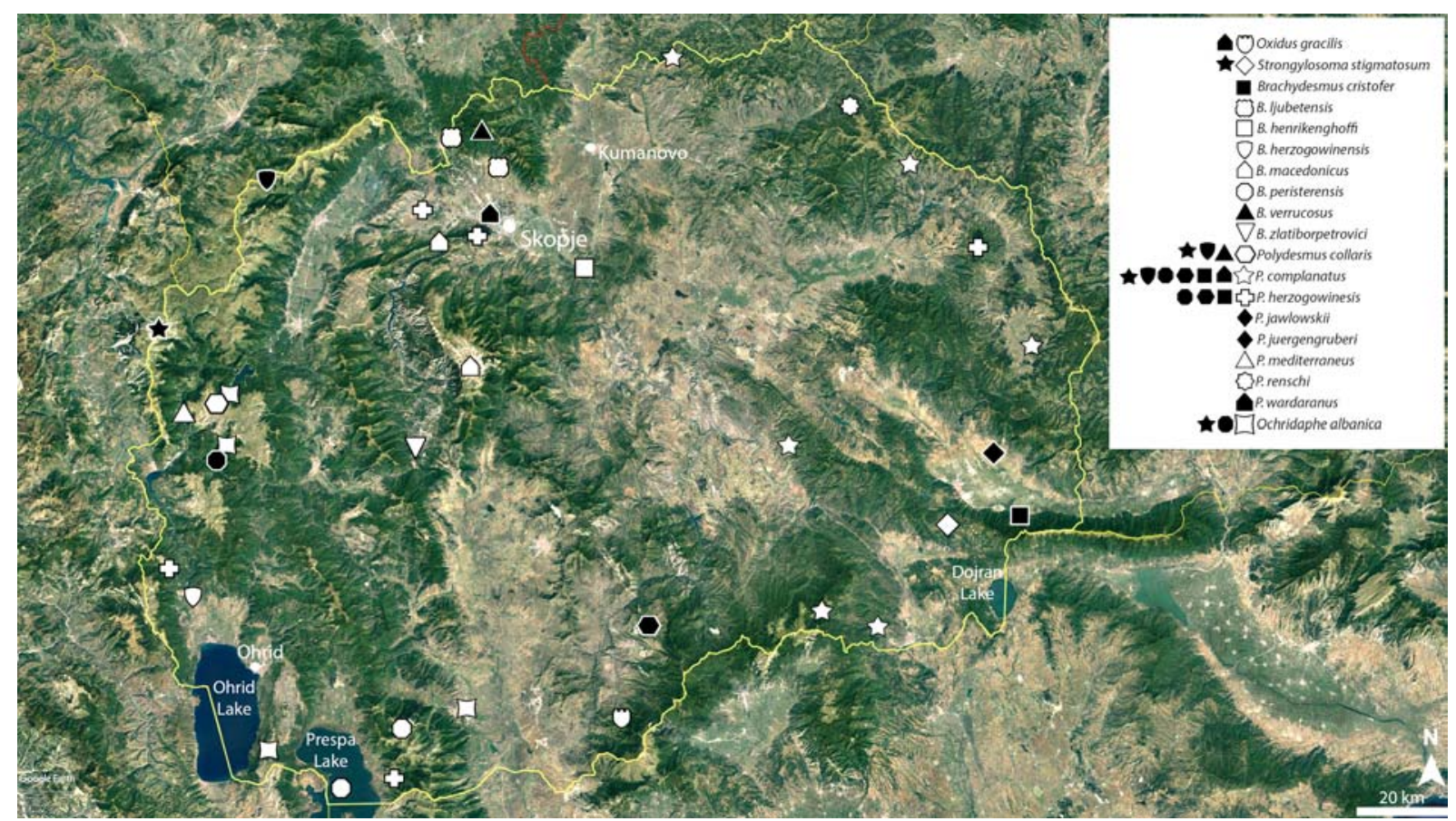

Fig. 5. Distribution of representatives of the order Polydesmida in North Macedonia.

Рис. 5. Распространение представителей отряда Polydesmida в Северной Македонии.

World distribution: Croatia, Republic of North Macedonia, and Serbia [Kime, Enghoff, 2017; Antić et al., 2018] Balkan endemic.

\section{Typhloiulus giganteus Ćurčić et Makarov, $2002 * *$}

MATERIAL. 1 , , Devina Cave, village of Deviè, Makedonski Brod, 10.III.2003, leg. E. Bušarova.

Distribution in the Republic of North Macedonia: Devina Cave, village of Devič, Makedonski Brod (present study); Momièek Cave, village of Belica, Makedonski Brod, Mt Dautica [Ćurčić et al., 2002b; Makarov et al., 2004] (Fig. 4).

North Macedonian endemic.

\section{Unciger foetidus (C.L. Koch, 1838)}

Julus foetidus C.L. Koch, 1838

Oncoiulus foetidus auct.

Distribution in the Republic of North Macedonia: Without precise locality [Mršić, 1993a; Makarov et al., 2004].

World distribution: Austria, Bosnia and Herzegovina, Croatia, Czech Republic, Danish mainland, Estonia, Finland, Germany, Great Britain, Hungary, Italian mainland, Lithuania, Latvia, Republic of North Macedonia, Norwegian mainland and inshore islands, Poland, Romania, Russia (Kaliningrad Region), Serbia, Slovakia, Slovenia, Sweden, Switzerland, the Netherlands, and Ukraine [Kime, Enghoff, 2017].

\section{Family NEMASOMATIDAE}

\section{Nemasoma varicorne C.L. Koch, 1847}

Isobates semisulcatus Menge, 1851

Isobates varicornis (C.L. Koch, 1847)
Distribution in the Republic of North Macedonia: Ljuboten, Šar Planina Mts [Attems, 1929]; without precise locality [Strasser, 1971a; Mršić, 1993a; Mauriès et al., 1997] (Fig. 4).

World distribution: Austria, Bosnia and Herzegovina, Belgium, Bulgaria, Belarus, Croatia, Czech Republic, Danish mainland, Finland, French mainland, Germany, Great Britain, Hungary, Ireland, Italian mainland, Lithuania, Luxembourg, Latvia, Northern Ireland, Norwegian mainland and inshore islands, Republic of North Macedonia, Poland, Romania, Russia (Kaliningrad Region, Central European Russia), Serbia, Sicily and neighbouring Italian islands, Slovakia, Slovenia, Sweden, Switzerland, the Netherlands, and Ukraine [Kime, Enghoff, 2011].

\section{Order POLYDESMIDA Family PARADOXOSOMATIDAE}

\section{Oxidus gracilis (C.L. Koch, 1847)}

Fontaria gracilis C.L. Koch, 1847

Paradesmus dasys Bollman, 1887

Kepolydesmus sontus Chamberlin, 1910

Distribution in the Republic of North Macedonia: Jelak, Šar Planina Mts [Ćurčić et al., 1999a]; Skopje [Mršić, 1993a; Makarov et al., 2004] (Fig. 5).

World distribution: Cosmopolitan, introduced worldwide [Kime, Enghoff, 2011].

\section{Strongylosoma stigmatosum (Eichwald, 1830)}

Iulus stigmatosus Eichwald, 1830

Strongylosoma pallipes Brandt, 1833

Tropisoma corrugatum C.L. Koch, 1847

Tropisoma ferrugineum C.L. Koch, 1847

Strongylosoma vejdovskyi Němec, 1895 
Strongylosoma balcanicum Schubart, 1934

Distribution in the Republic of North Macedonia: Crn Kamen, Mt Korab [Verhoeff, 1932]; Kosturino [Mršić, 1993a]; without precise locality [Attems, 1959; Strasser, 1971a; Mauriès et al., 1997; Makarov et al., 2004] (Fig. 5).

World distribution: Albania, Austria, Bosnia and Herzegovina, Bulgaria, Belarus, Croatia, Czech Republic, Germany, Hungary, Italian mainland, Lithuania, Latvia, Republic of North Macedonia, Moldova, Montenegro, Poland, Romania, Russia (Kaliningrad Region, Central European Russia, East Central Russia, Northwest Russia), Serbia, Slovakia, Slovenia, and Ukraine [Kime, Enghoff, 2011].

\section{Family POLYDESMIDAE}

\section{Brachydesmus cristofer Strasser, 1966*}

MATERIAL. $1 \sigma^{7}$, near the village of Popadija, Mt Belasica $1038 \mathrm{~m}$ a.s.1., Quercus-Fraxinus forest, 28.V.2010, leg. A.C. Gjorgjievska.

Distribution in the Republic of North Macedonia: near the village of Popadija, Mt Belasica (present study) (Fig. 5). donia.

Note: A species new to the Republic of North Mace-

World distribution: Bulgaria, Republic of North Macedonia [Kime, Enghoff, 2011; present study].

Balkan endemic.

\section{Brachydesmus ljubetensis Attems, 1912**}

Brachydesmus ljubotensis Mršić, 1985

Distribution in the Republic of North Macedonia: in front of Blatce Cave [Mršić, 1993a]; Ljuboten, Šar Planina Mts [Attems, 1912, 1929; Strasser, 1971a; Makarov et al., 2004]; without precise locality [Mauriès et al., 1997; Ćurčić et al., 2002a; Makarov et al., 2004] (Fig. 5).

World distribution: Republic of North Macedonia, Serbia [Kime, Enghoff, 2011].

Balkan endemic.

\section{Brachydesmus henrikenghoffi Mršić, 1993**}

Distribution in the Republic of North Macedonia: Katlanovo [Mršić, 1993b]; village of Sasa [Mršić, 1993a, b]; without precise locality [Ćurčić et al., 2002a; Makarov et al., 2004] (Fig. 5).

North Macedonian endemic.

\section{Brachydesmus herzogowinensis Verhoeff,} $1897 * *$

Brachydesmus armatus Strasser, 1933

Brachydesmus parvus Strasser, 1940

Brachydesmus septentrionalis Strasser, 1940

Brachydesmus confinis Strasser, 1969

Brachydesmus trifidus Strasser, 1973

Brachydesmus reflexus Strasser, 1975

Brachydesmus serbicus Mršić, 1988

MATERIAL. $1 \sigma^{7}, 4$ q+ , Mlečnik Cave, Tašmarunište, Struga, 21.IV.2006, leg. I. Karaman.

Distribution in the Republic of North Macedonia: Mlečnik Cave, village of Tašmarunište, Struga (present study); village of Sasa [Mršić, 1993a]; without precise locality [Mauriès et al., 1997; Makarov et al., 2004] (Fig. 5).

World distribution: Albania, Bosnia and Herzegovina, Bulgaria, Croatia, Republic of North Macedonia, Montenegro, Serbia, and Slovenia [Kime, Enghoff, 2011].
Balkan endemic.

\section{Brachydesmus macedonicus Mršić, 1988**}

MATERIAL. $1 \sigma^{\gamma}$, Ubava Cave, Matka, date and leg.: no data.

Distribution in the Republic of North Macedonia: Solunska Glava [Mršić, 1988b, 1993a]; Ubava Cave, Matka (present study); without precise locality [Ćurčić et al., 2002a; Makarov et al., 2004] (Fig. 5).

North Macedonian endemic.

\section{Brachydesmus peristerensis Verhoeff, 1932**}

Distribution in the Republic of North Macedonia: Mt Pelister [Strasser, 1971a]; Lake Prespa [Mršiæ, 1993a]; Virovi, Mt Pelister [Verhoeff, 1932; Attems, 1940; Mršić, 1993a]; without precise locality [Mauriès et al., 1997; Ćurčić et al., 2002a; Makarov et al., 2004] (Fig. 5).

World distribution: Bulgaria, Republic of North Macedonia [Kime, Enghoff, 2011].

Balkan endemic.

\section{Brachydesmus verrucosus Makarov et Antić,} $2013 * *$

Distribution in the Republic of North Macedonia: Skopska Crna Gora, near St. Ilija Monastery, 600 m [Antić et al., 2013] (Fig. 5).

North Macedonian endemic.

\section{Brachydesmus zlatiborpetrovici Ćurčić et} Makarov, 2002**

Distribution in the Republic of North Macedonia: Devina Cave, village of Devič, Makedonski Brod [Ćurčić et al., 2002a; Makarov et al., 2004] (Fig. 5).

North Macedonian endemic.

\section{Polydesmus collaris C.L. Koch, 1847}

Polydesmus rannensis Attems, 1895

Polydesmus tussilaginis Verhoeff, 1929

Polydesmus anienanus Verhoeff, 1932

Polydesmus salamandrinus Verhoeff, 1932

MATERIAL. $1 \sigma^{7}$, St. Ilija Monastery, Skopska Crna Gora, 600 m a.s.1., 14.IV.2006, leg. I. Karaman.

Distribution in the Republic of North Macedonia: Carevec, Mt Bistra [Mršić, 1988b, 1993a]; Crn Kamen, Mt Korab [Verhoeff, 1932; Attems, 1940]; Šar Planina Mts [Attems, 1940]; Skopska Crna Gora (present study) (Fig. 5).

World distribution: Albania, Austria, Bulgaria, Bosnia and Herzegovina, Croatia, Italian mainland, Hungary, Republic of North Macedonia, Montenegro, Romania, Serbia, and Slovenia [Bachvarova et al., 2017; Kime, Enghoff, 2011].

\section{Polydesmus complanatus (Linnaeus, 1761)}

Julus complanatus Linnaeus, 1761

Polydesmus constrictus Latzel, 1884

Polydesmus illyricus Verhoeff, 1893

Polydesmus balticus Verhoeff, 1907

Polydesmus fluviatilis Verhoeff, 1907

Polydesmus brohmeri Schubart, 1964

Polydesmus calaber Strasser, 1970

MATERIAL. 2 우, near the village of Popadija, Mt Belasica, 1038 m a.s.1., Quercus-Fraxinus forest, 28.V.2010; $1 \sigma^{7}$, same 
locality, $1100 \mathrm{~m}$ a.s.1., Festuca-Fagus community 28.V.2010; 1 $\mathrm{O}^{7}, 1$, same locality, $1200 \mathrm{~m}$ a.s.1., Calamintha-Fagus community, 28.V.2010; 1 \%, 1 \%, same locality, $847 \mathrm{~m}$ a.s.1., QuercusFraxinus forest, 3.VII.2010; 2 우, same locality, $1100 \mathrm{~m}$ a.s.1., Festuca-Fagus community, 3.VII.2010; $1 \sigma^{7}$, same locality, 1200 $\mathrm{m}$ a.s.1., Calamintha-Fagus community, 3.VII.2010; $4 \sigma^{7} \sigma^{7}$, same locality, $1038 \mathrm{~m}$ a.s.1., Quercus-Fraxinus forest, 4.X.2010; $3 \sigma^{7} \sigma^{7}$, same locality $1100 \mathrm{~m}$ a.s.1., Festuca-Fagus community, 4.X.2010; $4 \bigcirc^{7} \sigma^{7}, 1$ juv., same locality, $1200 \mathrm{~m}$ a.s.1., Calamintha-Fagus community, 4.X.2010; $1 \sigma^{7}, 5$ 0 , near the Pisana Skala slopes, Mt Belasica, $1442 \mathrm{~m}$ a.s.1., cleared area, 28.V.2010; $1 \sigma^{\top}, 1$ \% , and 1 juv., same locality, $1385 \mathrm{~m}$ a.s.1., Calamintha-Fagus community, 3.VII.2010, all leg. A.C. Gjorgjievska; 1 +, Mt Kožuf, 896 m a.s.1., Festuca-Fagus community, 19.VII.2016; 1 ア, Mt Kožuf, 181 m a.s.1., riparian habitat, Juglans-Platanus forest, 21.VII.2016, all leg. D. Matevski; $2 \sigma^{\top} \sigma^{\top}$, Lake Brezno, Šar Planina Mts, 6.IX.2017, leg. A.C. Gjorgjievska, S. Hristovski.

Distribution in the Republic of North Macedonia: Mt Belasica (present study); Bigor drop, Vitolište [Mršić, 1993a]; Lake Brezno, Šar Planina Mts (present study); Crn Kamen, Mt Korab [Verhoeff, 1932]; Mt Konečka [Mršić, 1993a]; Mt Kozjak, Vitolište [Mršić, 1993a]; Mt Kožuf (present study); Lazaropole [Mršić, 1993a]; Rateva Reka ravine [Mršić, 1993a]; village of Sasa [Mršić, 1993a]; Skopje [Mršić, 1993a]; without precise locality [Strasser, 1971a; Mauriès et al., 1997; Makarov et al., 2004] (Fig. 5).

World distribution: Albania, Asiatic Turkey, Austria, Bosnia and Herzegovina, Bulgaria, Belarus, Croatia, Czech Republic, Danish mainland, Estonia, European Turkey (without islands in the Sea of Marmara), Finland, Germany, Greek mainland, Hungary, Italian mainland, Lithuania, Latvia, Republic of North Macedonia, Moldova, Montenegro, Norwegian mainland and inshore islands, Poland, Romania, Russia (Kaliningrad Region, as well as Central European, East Central, North European, and Northwest Russia), Serbia, Slovakia, Slovenia, Sweden, Switzerland, Ukraine, and introduced into the Nearctic [Kime, Enghoff, 2011].

\section{Polydesmus herzogowinensis Verhoeff, 1897}

Polydesmus insulanus Attems, 1898

Polydesmus elchowensis Verhoeff, 1928

Polydesmus gladiatus Schubart, 1934

Polydesmus hercegowinensis auct.

MATERIAL. 1 J', Mt Golak, 1.VI.2000, leg. E. Stojkoska; 1 $\sigma^{7}, 1$, , between the localities of Pod and Suva Češma, Mt Belasica, $587 \mathrm{~m}$ a.s.1., Quercus-Carpinus forest, 28.V.2010; $2 \sigma^{7} \sigma^{\top}, 1$, near the village of Popadija, Mt Belasica, $847 \mathrm{~m}$ a.s.1., QuercusFraxinus forest, 28.V.2010; $9 \sigma^{\top} \sigma^{\top}, 1$, same locality, $1038 \mathrm{~m}$ a.s.1., Quercus-Fraxinus forest, 28.V.2010; $3 \sigma^{\top} \sigma^{\top}, 1$, same locality, $1100 \mathrm{~m}$ a.s.1., Festuca-Fagus community, 28.V.2010; 6 $\sigma^{7} \sigma^{7}$, same locality, $1300 \mathrm{~m}$ a.s.1., Calamintha-Fagus community, 28.V.2010; $1 \sigma^{7}$, same locality, $767 \mathrm{~m}$ a.s.1., Quercus-Fraxinus forest, 3.VII.2010; $1 \mathrm{O}^{\top}$, same locality, $847 \mathrm{~m}$ a.s.1., QuercusFraxinus forest , 3.VII.2010; $2 \sigma^{7} \sigma^{7}$, same locality, $1100 \mathrm{~m}$ a.s.1., Festuca-Fagus community, 3.VII.2010; 1 ๙ , same locality, 1200 $\mathrm{m}$ a.s.1., Calamintha-Fagus community, 3.VII.2010; 1 \%", same locality, $1100 \mathrm{~m}$ a.s.1., Festuca-Fagus community, 4.X.2010; 8 $\sigma^{7} \sigma^{7}$, near the Pisana Skala slopes, Mt Belasica, $1385 \mathrm{~m}$ a.s.1., Calamintha-Fagus community, 28.V.2010; 8 ○' $\sigma^{\top}, 2$ 우, same locality, $1442 \mathrm{~m}$ a.s.1., cleared area, 28.V.2010; $2 \sigma^{7} \sigma^{7}, 1$, same locality, $1442 \mathrm{~m}$ a.s.1., cleared area, 3.VII.2010; $1 \sigma^{\top}$, near Groba, Mt Belasica, $1300 \mathrm{~m}$ a.s.1., Calamintha-Fagus community, 3.VII 2010, all leg. A.C. Gjorgjievska; $1 \Im^{T}$, village of Brajčino, Mt Pelister, Fagus-Abies plantation, 1.VII.2016, leg. E. Džima.

Distribution in the Republic of North Macedonia: Mt Belasica (present study); village of Brajčino, Mt Pelister (present study); Mt Golak (present study); Jablanica [Verhoeff, 1932]; Kula, Kopanje [Ćurčić et al., 1999a; Makarov et al., 2004]; village of Lazaropole [Mršić, 1988b, 1993a]; village of Rašèe, near Skopje [Ćurčić et al., 1999a; Makarov et al., 2004]; village of Vitolište, Mt Kozjak [Mršić, 1988b, 1993a; Makarov et al., 2004]; Mt Vodno, Skopje [Attems, 1940] (Fig. 5).

World distribution: Albania, Asiatic Turkey, Bosnia and Herzegovina, Bulgaria, Croatia, Greek mainland, Republic of North Macedonia and Montenegro [Kime, Enghoff, 2011].

\section{Polydesmus jawlowskii Strasser, 1966**}

Distribution in the Republic of North Macedonia: Hamzali, Mt Ogražden [Mršić, 1993a] (Fig. 5).

World distribution: Bulgaria, Republic of North Macedonia [Kime, Enghoff, 2011].

Balkan endemic.

\section{Polydesmus juergengruberi Mršić, 1993**}

Distribution in the Republic of North Macedonia: Hamzali, Mt Ogražden [Mršić, 1993b]; without precise locality [Ćurčić et al., 2002a; Makarov et al., 2004] (Fig. 5).

North Macedonian endemic.

\section{Polydesmus mediterraneus Daday, 1889}

Polydesmus oertzeni Verhoeff, 1901

Polydesmus montenegrinus Attems, 1903

Polydesmus klisurensis Verhoeff, 1903

Polydesmus valachicus Tabacaru et Negrea, 1961

Polydesmus martensi Strasser, 1967

MATERIAL. $1 \sigma^{\Upsilon}$, Monastery of St. Jovan Bigorski, Mt Bistra, 13.IV.2006, leg. I. Karaman.

Distribution in the Republic of North Macedonia: $\mathrm{Mt}$ Bistra (present study); Skopje [Mršić, 1988b, 1993a] (Fig. 5). World distribution: Albania, Asiatic Turkey, Bulgaria, Croatia, European Turkey (without islands in the Sea of Marmara), Greek mainland, Republic of North Macedonia, Malta, Montenegro, Romania, Serbia, and Ukraine [Kime, Enghoff, 2011].

\section{Polydesmus renschi Schubart, 1934**}

Polydesmus tridens Attems, 1951

Polydesmus dobrogicus Ceuca, 1958

Polydesmus dobrogensis Tabacaru et Negrea, 1961

Distribution in the Republic of North Macedonia: village of Krklja [Mršić, 1993a] (Fig. 5).

World distribution: Bulgaria, Republic of North Macedonia, Romania, and Serbia [Antić et al., 2013a; Kime, Enghoff, 2011].

Balkan endemic.

\section{Polydesmus wardaranus Verhoeff, 1937**}

Distribution in the Republic of North Macedonia: Skopje [Verhoeff, 1937; Attems, 1940; Strasser, 1971a; Mršić, 1993a]; without precise locality [Mauriès et al., 1997; Ćurčić et al., 2002a; Makarov et al., 2004] (Fig. 5).

North Macedonian endemic.

\section{Family XYSTODESMIDAE}

\section{Ochridaphe albanica (Verhoeff, 1932)**}

Melaphe albanica Verhoeff, 1932 


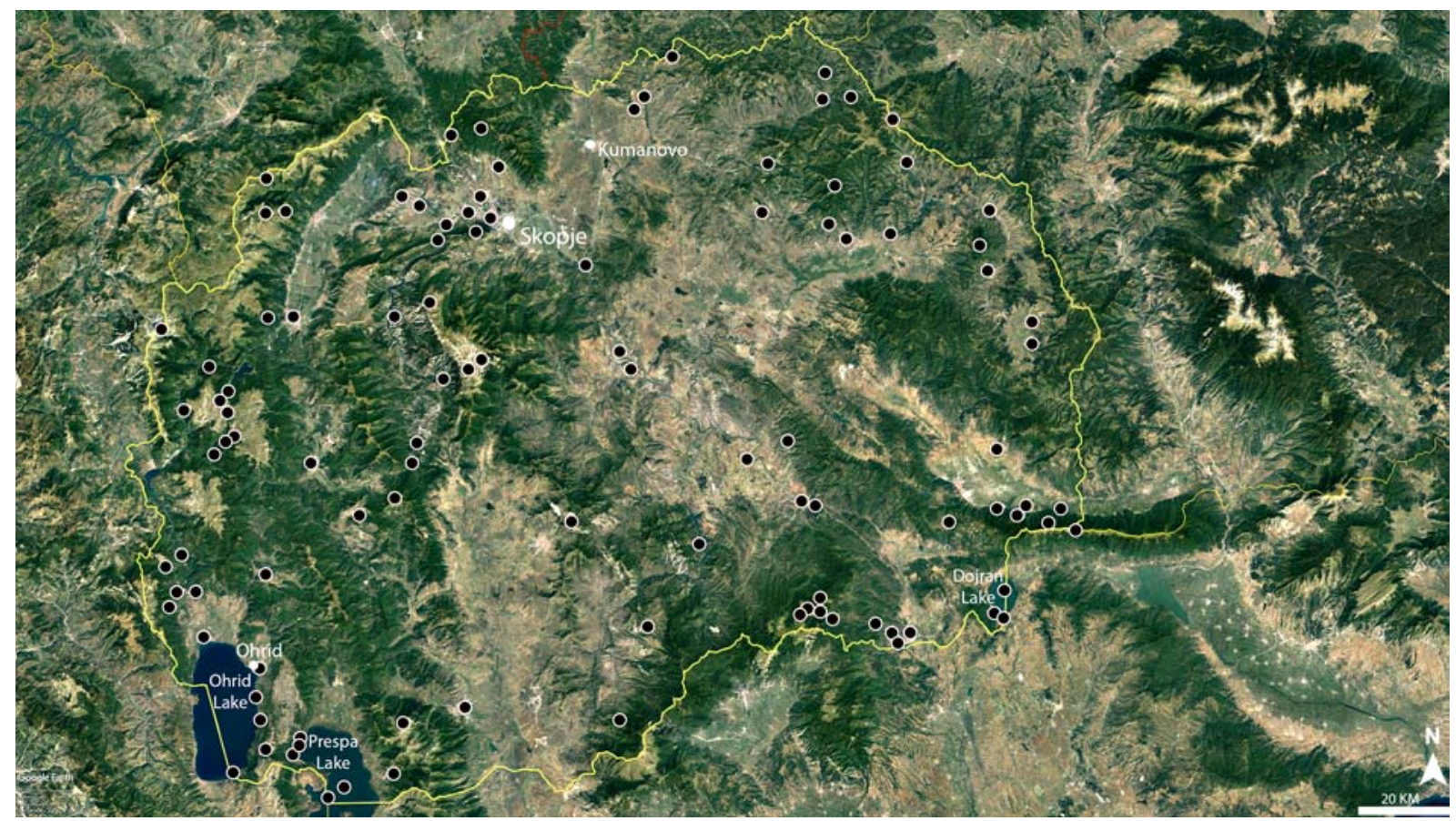

Fig. 6. Distribution of representatives of the class Diplopoda in North Macedonia.

Рис. 6. Распространение представителей класса Diplopoda в Северной Македонии.

Distribution in the Republic of North Macedonia: Begova Česma, Mt Pelister [Ćurčić et al., 1999a]; Mt Bistra, Mavrovo [Mršić, 1988b, 1993a]; Mt Galičica [Mršić, 1993a]; Mt Jablanica [Verhoeff, 1932; Strasser, 1971a]; Mt Korab [Verhoeff, 1932; Strasser, 1971a]; Lazaropole, Mt Bistra [Ćurčić et al., 1999]; Mala Reka, Mt Tresonče [Ćurčić et al., 1999a]; Mavrovo [Ćurčić et al., 1999a]; without precise locality [Mauriès et al., 1997; Ćurčić et al., 2002a; Makarov et al., 2004] (Fig. 5).

World distribution: Albania, Republic of North Macedonia, and Greek mainland [Kime, Enghoff, 2011].

Balkan endemic

\section{Discussion}

The millipede fauna of North Macedonia consists of 67 species, 33 genera, 15 families, and seven orders (Fig. 6). The best represented are the species-rich families Julidae, with 29 species, and Polydesmidae, with 16 species. The genera Megaphyllum Verhoeff, 1894, Polydesmus Latreille, 1802, and Brachydesmus Heller, 1858 contribute eight species each. Taxa new for the diplopod fauna of North Macedonia are Hirudisoma hirsutum (Verhoeff, 1901) and Brachydesmus cristofer Strasser, 1966. Hirudisoma hirsutum, as a representative of a genus and family (Hirudisomatidae) new to North Macedonia, is found in the neighbouring countries of Bulgaria and Greece, as well as in Turkey. Brachydesmus cristofer has so far been recorded only in Bulgaria. After the revision of Vagalinski \& Lazányi [2018], two species, Megaphyllum crassum (Attems, 1929) and M. imbecillum (Attems, 1935), are presented as combinatio nova, Italoiulus crassus (Attems, 1929) and Graecoiulus imbecillus (Attems, 1935), re- spectively. Thirty one endemics comprise ca. $46 \%$ of the North Macedonian millipede fauna, with 12 species being endemic to North Macedonia and 19 considered as Balkan endemics.

With its complex geological history, variety of natural conditions, various biogeographical influences, and vast number of endemic and relict species, the Balkan Peninsula is a heterogeneous and diverse region. During the last Ice Age, the Balkan Peninsula represented one of the three main refugia of Europe, the other two being the Iberian and Italian Peninsulas, where a great number of relict and endemo-relict species and communities were preserved in various epigean and subterranean habitats [Savić, 2008]. In some neighbouring countries, the reported number of species is well over 100 (nearly 200 in Croatia), although this is not the case in Albania and Montenegro, where it is in the range of currently known diversity of the North Macedonian fauna [Antić et al., 2013a]. It can therefore be presumed that the millipede fauna of North Macedonia is probably more abundant than has been shown thus far.

Acknowledgements. This work was supported by the Serbian Ministry of Education, Science, and Technological Development (Grant No. 173038). First of all, we are most grateful to Sergei I. Golovatch for his suggestions to improve the manuscript. The authors are highly grateful to all the collectors: Emilija Stojkoska, Snežana Stanković, Ivo Karaman, Radmila Blaževska, Dragan Matevski, Marjan Komnenov, Slavèo Hristovski, Angela Taseska, Dalibor Stojanović, and Elena Džima. The distribution maps were prepared with the help of Nemanja Jovanović. Finally, the authors would like to thank Mr. Raymond Dooley for correcting the English version of the manuscript. 


\section{References}

Antić D.Ž., Curčić B.P.M., Tomić V.T., Rađa T., Rađa B., Milinčić MA., Makarov S.E. 2013. Two new species of Brachydesmus Heller, 1858 from the Balkan Peninsula (Diplopoda: Polydesmida: Polydesmidae) // Archives of Biological Sciences. Vol.65. No.3. P.1233-1243.

Antić D.Ž., Ćurčić B.P.M., Tomić V.T., Ćurčić S.B., Stojanović D.Z., Dudić B.D., Makarov S.E. 2013a. One hundred millipede species in Serbia (Arthropoda: Myriapoda: Diplopoda) // Archives of Biological Sciences. Vol.65. No.4. P.1559-1578.

Antić D.Ž., Dražina T., Rađa T., Lučić L.R., Makarov S.E. 2018. Review of the genus Typhloiulus Latzel, 1884 in the Dinaric region, with a description of four new species and the first description of the male of Typhloiulus insularis Strasser, 1938 (Diplopoda: Julida: Julidae) // Zootaxa. Vol.4455. No.2. P.258294.

Attems C. 1927. Über palaearktische Diplopoden // Archiv für Naturgeschichte. Bd.92. H.1-2. S.1-256.

Attems C. 1929. Die Myriopodenfauna von Albanien und Jugoslavien // Zoologische Jahrbücher, Abteilung für Systematik, Ökologie und Geographie der Tiere. Bd.56. S.269-356.

Attems C. 1940. Polydesmoidea. III // Das Tierreich. Lfg.70. S.1577.

Attems C. 1959. Die Myriapoden der Höhlen der Balkanhalbinsel. Nach dem Material der "Biospeologica Balcanica" // Annalen des Naturhistorischen Museums in Wien. Bd.63. S.281-406.

Bachvarova D., Vagalinski B., Doichinov A., Stoev P. 2017. New records of millipedes and centipedes from Bulgaria, with an annotated checklist of the Bulgarian myriapods // Zootaxa. Vol.4263. No.3. P.507-526.

Ceuca T. 1992. Quelques aspects sur la faunistique, l'écologie et la zoogéographie des diplopodes de la région Balkanique // $\mathrm{Ad}-$ vances in Myriapodology, Berichte des Naturwissenschaftlichmedizinischen Vereins in Innsbruck. Suppl.10. No.465. P.411429.

Ćurčić B.P.M., Makarov S.E., Karaman I.M., Dimitrijević R.N., Ćurčić S.B. 1999a. Some comments on the diplopods (Diplopoda, Myriapoda) from Yugoslavia. Part I - Glomerida // Archives of Biological Sciences. Vol.51. P.11-12.

Ćurčić B.P.M., Makarov S.E., Karaman I.M., Dimitrijević R.N., Ćurčić S.B. 1999b. Some remarks on the millipede fauna of Macedonia // Archives of Biological Sciences. Vol.51. P.17-18

Ćurčić B.P.M., Makarov S.E., Stanković-Jovanović S.V. 2002a. Taksonomske implikacije endemiène diferencijacije Diplopoda (Myriapoda) u Jugoslaviji i Makedoniji // Zbornik radova Odbora za kras i speleologiju VII. Beograd: Srpska akademija nauka i umetnosti. P.93-102 [in Serbian].

Ćurčić B.P.M., Makarov S.E., Stanković-Jovanović S.V., Boškova T. 2002b. Istorijski razvoj geografske distribucije nekih peæinskih diplopoda (Myriapoda) u Jugoslaviji i Makedoniji // Zbornik radova Odbora za kras i speleologiju VII. Beograd: Srpska akademija nauka i umetnosti. P.103-112 [in Serbian].

Golovatch S.I., Kime R.D. 2009. Millipede (Diplopoda) distributions: A review // Soil Organisms. Vol.81. No.3. P.565-597.

Hopkin S.P., Read H.J. 1992. The Biology of Millipedes. Oxford: Oxford University Press. $246 \mathrm{pp}$

Kime R.D., Enhoff H. 2011. Atlas of European Millipedes (Class Diplopoda), Vol.1 - Orders Polyxenida, Glomerida, Platydesmida, Siphonocryptida, Polyzoniida, Callipodida, Polydesmida // Pensoft Series: Fauna Europeaea Evertebrata ${ }^{\top} 3$. Sofia-Moscow: Pensoft Publishers. 282 pp.

Kime R.D., Enghoff H. 2013. Fauna Europaea: Chordeumatidae. Museum für Naturkunde Berlin, online at: https://fauna-eu.org, version 2017.09 (accessed on 4 September 2018)

Kime R.D., Enghoff H. 2017. Atlas of European millipedes 2: Order Julida (Class Diplopoda) // European Journal of Taxonomy. Vol.346. P.1-299.

Lazányi E., Vagalinski B., Korsós Z. 2012. The millipede genus Megaphyllum Verhoeff, 1894 in the Balkan Peninsula, with description of new species (Myriapoda: Diplopoda: Julida: Julidae) // Zootaxa. Vol.3228. No.1. P.1-47.
Makarov S.E. 2001. Schizmohetera curcici, a new cavernicolous species from Macedonia (Diplopoda, Chordeumatida, Neoatractosomatidae) // Archives of Biological Sciences. Vol.53. P.19-20.

Makarov S.E., Mitić B.M., Lučić L.R., Tomić V.T., Ćurčić S.B. 2003. Two new glomeridellids (Glomeridellidae, Diplopoda) from Montenegro and Macedonia // Periodicum Biologorum. Vol.105. No.4. P.473-477.

Makarov S.E., Ćurčić B.P.M., Tomić V.T., Legakis A. 2004. The diplopods of Serbia, Montenegro, and the Rebublic of Macedonia. Belgrade: Institute of Zoology, Faculty of Biology, University of Belgrade. $440 \mathrm{pp}$.

Mauriès J.-P., Golovatch S.I., Stoev P.E. 1997. The millipedes of Albania: recent data, new taxa; systematical, nomenclatural and faunistical review (Myriapoda, Diplopoda) // Zoosystema. Vol.19. Nos 2-3. P.255-292.

Mauriès J.-P. 2003. Schizmohetera olympica sp. n. from Greece, with a reclassification of the superfamily Neoatractosomatoidea (Diplopoda: Chordeumatida) // Arthropoda Selecta. Vol.12. No.1. P.9-16.

Mršić N. 1987. Genus Xestoiulus Verhoeff, 1893 (Diplopoda: Julidae) in Yugoslavia // Razprave IV Razreda. Ljubljana: Slovenska akademija znanosti in umetnosti. Vol.28. P.3-25.

Mršić N. 1988a. Intereleptoiuus cernagoranus g.n., sp.n. (Diplopoda: Julidae) and a survey of the tribus Leptoiulini in Yugoslavia // Biološki Vestnik. Vol.36. P.31-52.

Mršić N. 1988b. Polydesmida (Diplopoda) of Yugoslavia. I // Academia Scientiarum et Artium Slovenica, Classis IV, Historia Naturalis. Vol.29. P.69-112.

Mršić N. 1993a. The fauna of diplopods (Diplopoda) of Macedonia // Razprave IV Razreda. Ljubljana: Slovenska akademija znanosti in umetnosti. Vol.34. P.19-44.

Mršić N. 1993b. Polydesmus (Nomarchus) juergengruberi n.sp. and Brachydesmus (Brachydesmus) henrikenghoffi n.sp. (Polydesmida, Diplopoda) from Macedonia // Zoologischer Anzeiger. Vol.232. P.177-180.

Petkovski S. 2009. National Catalogue (Check List) of Species. Ref. UNDP Contract: Biodiversity and Protected Areas Consultant (National) within the Project, 58373.

Savić I.R. 2008. Diversification of the Balkan fauna: its origin, historical development and present status // Advances in Arachnology and Developmental Biology. Vol.12. P.57-78.

Sierwald P., Bond J.E. 2007. Current status of the myriapod class Diplopoda (millipedes): taxonomic diversity and phylogeny // Annual Review of Entomology. Vol.52. P.401-420.

Spelda J. 2013. SysMyr: Systematic myriapod database. Zoologische Staatssammlung München, online at http://www.gbifev2. mwn.de/GloMyrIS/searchh_myr.htm, version February 2013 (accessed on 4 September 2018).

Strasser K. 1962. Die Typhloiulini (Diplopoda, Symphyognatha) // Atti del Museo Civico di Storia Naturale di Trieste. T.23. P.1-77.

Strasser K. 1971a. Diplopoda // Catalogus Faunae Jugoslaviae Consilium Academiarum Scientiarum Rei Publicae Socialisticae Foederative Jugoslaviae. Academia Scientiarum et Artium Slovenica. T.3. No.5. P.1-50.

Strasser K. 1971b. Über Diplopoden Jugoslawiens // Senckenbergiana Biologica. Bd.52. S.313-343.

Strasser K. 1976. Über Diplopoda-Chilognatha Greiechenlands, II // Revue Suisse de Zoologie. T.83. Fasc.3. P.579-645.

Vagalinski B., Stoev P. Enghoff H. 2015. A review of the millipede genus Typhloiulus Latzel, 1884 (Diplopoda: Julida: Julidae), with a description of three new species from Bulgaria and Greece // Zootaxa. Vol.3999. No.3. P.334-362.

Vagalinski B., Lazányi E. 2018. Revision of the millipede tribe Brachyiulini Verhoeff, 1909 (Diplopoda: Julida: Julidae), with descriptions of new taxa // Zootaxa. Vol.4421. No.1. P.1-142.

Verhoeff K.W. 1923. Zur Kenntnis der Palästina-Chilognathen und über einige andere mediterrane Formen // Archiv für Naturgeschichte. Bd.89. S.112-157.

Verhoeff K.W. 1932. Diplopoden-Beiträge // Zoologische Jahrbücher, Abteilung für Systematik, Ökologie und Geographie der Tiere. Bd.62. H.5-6. S.469-666.

Responsible editor S.I. Golovatch 